\title{
Ehe und Partnerschaft im Recht der Europäischen Union - Wie weit reicht die Bestimmungsmacht der Mitgliedstaaten
}

\author{
Dagmar Richter*
}

Inhalt

A. Einführung 302

I. Problembeschreibung 302

II. Begriffe: „Geschlecht“ und „sexuelle Orientierung“ 303

III. Regelungsmodelle der europäischen Staaten im Überblick Gibt es einen europäischen Konsens? 304

B. Rechtliche Grundlagen $\quad 307$

I. Kompetenzverteilung zwischen EU und Mitgliedstaaten 307

1. Kompetenz der EU zur Bekämpfung von Diskriminierung 307

2. Kompetenz der Mitgliedstaaten zur Regelung des Familienstands und davon abhängiger Leistungen $\quad 307$

3. Der Grundsatz der Einheitlichkeit der persönlichen Rechtsstellung 308

II. Das Verbot der Diskriminierung aufgrund der sexuellen Orientierung 309

1. Die Richtlinie zur Festlegung eines allgemeinen Rahmens für die Verwirklichung der Gleichbehandlung in Beschäftigung und Beruf $\quad 309$

2. Das Verbot der Diskriminierung aufgrund sexueller Orientierung als Grundrecht der EU (Art. 6 EUV; Art. 21 GrCh; Art. 14 in Verbindung mit Art. 8 EMRK)

III. Recht auf Ehe aus Art. 6 EUV in Verbindung mit Art. 9 GrCh, Art. 12 EMRK? 311

IV. Anerkennung des Partnerschafts- oder Ehestatus in anderen EU-Ländern? 312

1. Der Ehebegriff des europäischen Verfahrensrechts 312

2. Gleichgeschlechtliche Lebens- oder Ehepartner als „Familienangehörige“ 314

a) Regelungsstrategien der Union: Aufenthaltsrichtlinie und Familienzusammenführungsrichtlinie im Lichte grundrechtlicher Grenzen

b) Begriff des „Ehegatten” im Sekundärrecht und Öffnung der Ehe in einzelnen Mitgliedstaaten

* Prof. Dr. iur. Dagmar Richter ist Professorin und Mitglied des Instituts für Rechtswissenschaften der Polnischen Akademie der Wissenschaften (INP PAN) in Warschau. Der Beitrag geht auf einen Vortrag zurück, den die Autorin am 7.10.2013 an der FernUniversität Hagen gehalten hat. 
C. Rechtspraxis 318

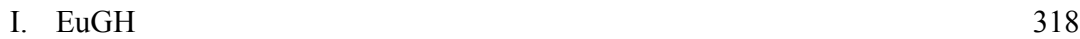

1. Ausgewählte Fälle 318

a) D. und Königreich Schweden (2001) 318

b) Maruko (2008) 318

c) Dittrich, Klinke und Müller (2012) 321

2. Bewertung: Fortschreitender Einfluss des Europarechts auf die Rechtsordnungen der Mitgliedstaaten

II. EGMR: Schalk und Kopf (2010) 322

D. Umsetzungsprobleme - am Beispiel des deutschen Rechts 324

I. Aufgabe des Abstandsgebots und Konservierung eines normativen Ehebegriffs

II. Begrenzung des Erfordernisses der Verschiedengeschlechtlichkeit auf den Zeitpunkt des Eheschlusses

III. Fehlen eines gesetzgeberischen Konzepts und „scheibchenweiser“ Nachvollzug des Europarechts

E. Analyse und Prognose: Ist die Öffnung der Ehe unausweichlich? 330

I. Problem- und Entwicklungsdimensionen 330

1. Gleichgeschlechtliche Ehe und Zukunft der Gesellschaft 330

2. Die grundrechtliche Dimension 331

3. Die Binnenmarktdimension 332

II. Zukunftsszenario: Entstehung einer „Ehe zweiter Klasse“? 333

\section{A. Einführung}

\section{Problembeschreibung}

Das Recht der Europäischen Union verbietet in seinem Anwendungsbereich jede Diskriminierung aufgrund der sexuellen Orientierung; doch fallen Regelungen zum Familienstand und davon abhängigen Leistungen in die ausschließliche Zuständigkeit der Mitgliedstaaten. ${ }^{1}$ Einerseits entscheiden also die Mitgliedstaaten allein, in welcher Form gleichgeschlechtliche Partner eine dauerhafte Rechtsbeziehung zwischen sich begründen können, ob in Form einer „Ehe“, „Lebenspartnerschaft" oder etwas Anderem. Andererseits gilt das Verbot der Diskriminierung aufgrund der sexuellen Orientierung europaweit. Zwischen Beidem besteht ein Spannungsverhältnis, da die Verweisung von Menschen mit seltenen Ausprägungen der sexuellen Orientierung auf nicht-eheliche Verbindungsformen den Keim der Diskriminierung in sich trägt. Das lehren jedenfalls die Erfahrungen mit ,separate

1 Eingehend unter B.I.1.und B.II. Einen Überblick geben Wolff, Ehe und Familie in Europa, EuR 2005, S. 721 ff.; Jakob, Die eingetragene Lebenspartnerschaft im Europarecht, FamRZ 2002, S. $501 \mathrm{ff}$. 
but equal"-Doktrinen ${ }^{2}$ auf anderen Feldern der Unterscheidung anhand gruppenbildender Merkmale: Weil die Einrichtungen der normprägenden Mehrheit (z.B. Ehe) in der sozialen Wirklichkeit als von vornherein prestigeträchtiger als die der Minderheit (z.B. gleichgeschlechtliche Partnerschaft) gelten, ist tatsächliche Gleichbehandlung so nicht herzustellen. Dieser Beitrag wird der Frage nachgehen, wie weit das Bestimmungsrecht der Mitgliedstaaten reicht und ob es im europäischen Rechtsraum nicht unvermeidlich zu einer Angleichung der Formen von Partnerschaft bis hin zur Öffnung der Ehe für Homosexuelle kommen muss.

\section{Begriffe: „Geschlecht“" und „sexuelle Orientierung““}

Eine Schlüsselfrage lautet: Warum wird die Problematik der Benachteiligung homosexueller Menschen eigentlich nicht unter dem Kriterium des „Geschlechts“ behandelt und Diskriminierung auf dieser Grundlage verboten ${ }^{3}$ Unter „Geschlecht“ versteht die medizinische Wissenschaft inzwischen eine Fülle von Ausprägungen im chromosomalen, gonadalen, genitalen, psychischen und sozialen Sinne. ${ }^{4}$ Fallen diese Parameter auseinander, liegt eine ungewöhnliche bzw. seltene Form der sexuellen Orientierung ${ }^{5}$ bzw. der sexuellen Identität ${ }^{6}$ vor, zum Beispiel Homosexualität, Intersexualität oder Transsexualität, ${ }^{7}$ heute zunehmend in Kürzeln wie „LGBT“ bzw. „LGBTI“ (lesbische, schwule, bi-, trans- und intersexuelle Personen) versteckt. ${ }^{8}$ So gesehen erscheinen die uns geläufigen Formen des männlichen und weiblichen Geschlechts als nichts Anderes als die am meisten verbreiteten Formen bzw. Unterformen der „sexuellen Orientierung“, während weniger bzw. selten verbreitete Formen selbstverständlich auch Ausprägungen der „Geschlechtlichkeit“ sind. Das Geschlecht eines Menschen ist nicht nur viel weniger eindeutig zweigeteilt als tradi-

2 Grundlegend dazu die Rechtsprechung des U.S. Supreme Court zur Vereinbarkeit der Rassentrennung mit dem 14. Amendment zur U.S.-Verfassung aufgrund der ,separate but equal doctrine“" Noch befürwortend: 163 U.S. 537 (1896) - Plessy v. Ferguson; erstmals verwerfend: 347 U.S. 483 (1954) - Brown v. Board of Education.

3 Siehe Adamietz, Geschlecht als Erwartung - Das Geschlechtsdiskriminierungsverbot als Recht gegen Diskriminierung wegen der sexuellen Orientierung und der Geschlechtsidentität, 2011.

4 Psychrembel, Klinisches Wörterbuch, 265. Aufl. 2014, Stichwort „Geschlecht“.

5 „Sexuelle Orientierung“ und ,sexuelle Identität“ werden teilweise synonym gebraucht, teilweise auch streng voneinander unterschieden, etwa in dem Sinne, dass die sexuelle Orientierung als Ausrichtung auf bestimmte Sexualpartner einen bloßen Unterfall der sexuellen Identität darstellen soll. Völlig fehl geht die von der Bundesregierung mitgeteilte Behördenpraxis, Homosexuelle und Transsexuelle mit der Kategorie der Sexualstraftäter zu vermengen. Siehe BT-Drs. 18/343 v. 24.1.2014, S. 7.

6 Siehe Hohmann-Dennhardt, Gleichheit nur für Heteros? - Keine Diskriminierung wegen der sexuellen Identität, KJ 2009, Beiheft 1, S. 125 ff.; sowie die Beiträge im Sonderheft „Geschlechtsidentität“ in: Aus Politik und Zeitgeschichte (Beilage zu „Das Parlament“), 2012, Heft 20-21.

7 Eingehend Richter, in: Merten/Papier (Hrsg.), Handbuch der Grundrechte in Deutschland und Europa, Bd. V, 2013, Gleichberechtigung von Mann und Frau, § 126, S. $951 \mathrm{ff}$.

8 Das gilt insbes. auch für EU-Dokumente. Siehe z.B. Entwurf einer Entschließung des Europäischen Parlaments zu dem EU-Fahrplan zur Bekämpfung von Homophobie und Diskriminierung aus Gründen der sexuellen Orientierung und der Geschlechtsidentität (2013/2183(INI)) v. 8.1.2014, www.europarl.europa.eu/sides/getDoc.do?type $=$ REPORT \&reference $=$ A7-2014-0009\&language $=\mathrm{DE}(25.6 .2014)$. 
tionell angenommen, sondern in extremen Fällen überhaupt nicht mehr eindeutig feststellbar. $^{9}$

Rechtswissenschaft und -praxis hinken diesen Erkenntnisfortschritten immer noch hinterher. Zwar sehen sie im Geschlecht heute mehr als eine nur ,äußere körperliche Beschaffenheit" 10 und nehmen zum Beispiel auch Rollenzuschreibungen in den Blick; ${ }^{11}$ auch solche Erweiterungen des Geschlechtsbegriffs bleiben jedoch immer am „dual-bipolaren Schema“ des typisch (= heterosexuell) Männlichen oder typisch Weiblichen haften und bilden die Wirklichkeit so nur unvollkommen ab. ${ }^{12}$ Das ist normativ auch so gewollt; denn würde zum Beispiel Homosexualität als eine Erscheinungsform des Geschlechts angesehen, wären die Regelungen zur Gleichbehandlung bzw. Gleichstellung von Männern und Frauen anwendbar. Dies verfehlt augenscheinlich den Sinn und Zweck dieser Normen, denn Homosexuelle werden nicht deshalb benachteiligt, weil sie Frau oder Mann sind, sondern weil sie dem Rollenbild der typischen Frau oder des typischen Mannes aufgrund ihrer ungewöhnlichen sexuellen Orientierung nicht entsprechen.

Nun könnte man aber einwenden, dass die Nichterfüllung einer mit dem Geschlecht verbundenen Rollenerwartung gerade auch ein Fall der geschlechtsbedingten Bewertung ist, aus der dann folgerichtig eine Diskriminierung aufgrund des Geschlechts erwachsen kann. Zumindest könnte man Regelungen, die an das „Geschlecht“ insgesamt und nicht explizit an „Männer“ und „Frauen“ anknüpfen, so verstehen. Solche Konsequenzen werden aber weder im Verfassungs- noch im Europa-, noch im Völkerrecht gezogen. Einsam steht hier die Entscheidung des U.N. Human Rights Committee im Fall Toonen, in dem die Diskriminierung Homosexueller tatsächlich einmal als Diskriminierung aufgrund des Geschlechts qualifiziert worden war. ${ }^{13}$ Diese Bewertung konnte sich aber selbst in diesem Gremium nicht dauerhaft etablieren, weil sie keinen Rückhalt in der Staatenpraxis fand. Soweit sich staatliche Rechtsordnungen überhaupt zu dieser Frage positionieren, entscheiden sie sich stets für die Schaffung einer gesonderten Kategorie der „sexuellen Orientierung“ neben dem Geschlecht. So verhält es sich auch im Recht der Europäischen Union.

\section{Regelungsmodelle der europäischen Staaten im Überblick - Gibt es einen europäischen Konsens?}

In Europa gibt es bislang (Stand: März 2014) zehn Staaten, die die Ehe für gleichgeschlechtliche Paare geöffnet haben: Auf die Niederlande (Gesetz vom 19. Dezember 2000/Inkrafttreten: 1. April 2001) als erstes Land der Welt folgten Belgien (30. Januar 2003/1. Juni

9 Siehe jetzt $\S 22$ Abs. 3 PStG: „Kann das Kind weder dem weiblichen noch dem männlichen Geschlecht zugeordnet werden, so ist der Personenstandsfall ohne eine solche Angabe in das Geburtenregister einzutragen." Einführung durch Art. 1 (6b) Personenstandsrechts-Änderungsgesetzes v. 7.5.2013, BGB1. 2013 I, 1122; Inkrafttreten am 1.11.2013 (Art. 10 Abs. 2 PStRÄndG).

10 So z.B. noch KG Berlin, NJW 1965, 1084 (1084).

11 Grundlegend BVerfGE 85, 191 (208 ff.) - Nachtarbeitsverbot.

12 Siehe Richter, (Fn. 7), S. 951, Rdnr. 12.

13 Communication No. 488/1992 (CCPR/C/50/D/488/1992) - Toonen v. Australia (31.3.1994), abgedruckt in: United Nations High Commissioner for Human Rights, International Covenant on Civil and Political Rights, Selected Decisions of the Human Rights Committee under the Optional Protocol, vol. 5 (47th to 55th session), 2005, S. $133 \mathrm{ff}$. 
2003), Spanien (30. Juni 2005/3. Juli 2005), Norwegen (17. Juni 2008/1. Januar 2009), Schweden (1. April 2009/1. Mai 2009), Portugal (Februar 2010/5. Juni 2010), Island (11. Juni 2010/ 27. Juni 2010), Dänemark (7. Juni 2012/15. Juni 2012), Frankreich (23. April 2013/ 17. Mai 2013) sowie England und Wales (17. Juli 2013/Sommer 2014). ${ }^{14}$ Dabei handelt es sich in Bezug auf die 47 Staaten des Europarates um ein knappes Fünftel der Staaten. Von den zehn aufgeführten Staaten gehören acht Staaten der EU an. Sie stellen mehr als ein Viertel der insgesamt 28 Mitgliedstaaten.

Unterhalb der Ehe sehen 14 Staaten die eingetragene Partnerschaft und sieben weitere Staaten vergleichbar registrierte Lebensformen vor; Ungarn und Kroatien erkennen gleichgeschlechtliche Lebenspartnerschaften ohne behördliche Eintragung an. Staaten, welche die Ehe geöffnet haben, behalten zumeist die zuvor eingeführte Form der eingetragenen Partnerschaft bei. Klare Erklärungsmuster für eine eher liberale oder eher restriktive Haltung gibt es nicht. Die politische Ausrichtung der jeweiligen Regierung scheint aber eine größere Rolle als zum Beispiel der Einfluss der katholischen Kirche zu spielen. So haben beispielsweise Spanien unter der sozialistischen Regierung Zapatero die Ehe geöffnet ${ }^{15}$ und Irland 2010/2011 ein Gesetz über die Civil Partnership in Kraft gesetzt. ${ }^{16}$ Andererseits hat in Polen die Debatte über die Einführung der eingetragenen Partnerschaft die Regierung Tusk vor eine Zerreißprobe gestellt, da der konservativ-katholische Flügel der eigenen Partei (Bürgerplattform) wie auch der amtierende Justizminister sich entsprechenden Gesetzesvorhaben verweigerten. ${ }^{17}$

Soweit sich die Staaten für Partnerschaftsgesetze entschieden haben, lassen sich zwei sehr unterschiedliche Grundansätze ausmachen: Zum einen gibt es das Modell der nordischen Staaten, welche die eingetragene Partnerschaft grundsätzlich der Ehe gleichstellen und nur ausgesuchte Materien im Rahmen einer „Negativliste” davon ausnehmen. Davon unterscheidet sich das Verweisungsmodell, wie es zum Beispiel Deutschland praktiziert, wo explizit auf einzelne anwendbare Ehevorschriften verwiesen wird. Während die nordischen Staaten bewusst darüber entscheiden, welche Begünstigungen der Ehe sie den homosexuellen Paaren vorenthalten wollen und sich die politische Mehrheit dafür demokratisch verantworten muss, entzieht sich der deutsche Gesetzgeber im Lebenspartner-

14 Siehe Europa-Übersicht der Homosexuellen Initiative (HOSI) Wien, www.hosiwien.at/ueber-uns/ archiv/europa-ubersicht/ (25.6.2014). Zitate der niederländischen, belgischen und spanischen Regelungen (mit Nachweis und Übersetzung) bietet Winklerv. Mohrenfels, Die gleichgeschlechtliche Ehe im deutschen IPR und im europäischen Verfahrensrecht, in: Arkan/Yongalik (eds.), Liber Amicorum Tuğrul Ansay, 2006, S. 528 f. Siehe auch EU Agency for Fundamental Rights, Länderberichte (Thematic Studies), Dezember 2010, http://fra.europa.eu/en/national-contribution/2012/ country-thematic-studies-homophobia-transphobia-and-discrimination (25.6.2014).

15 Ley 13/2005 v. 1.7.2005, Boletin Oficial del Estado (BOE), Nr. 157 v. 2.7.2005, S. 23632, änderte insoweit die Definition der Ehe in Art. 44 des Zivilgesetzbuches (Código Civil). Wortlaut mit Nachweis und Übersetzung bei Winkler v. Mohrenfels, (Fn. 14), S. 529 f. Zur VerfassungsmäBigkeit des Gesetzes: Tribunal Constitucional de España, Sentencia 198/2012 v. 6.11.2012, http:// hj.tribunalconstitucional.es/en/Resolucion/Show/23106 (25.6.2014).

16 Civil Partnership and Certain Rights and Obligations of Cohabitants Act 2010, No. 24 of 2010, www.irishstatutebook.ie/2010/en/act/pub/0024/sec0005.html\#sec5 (25.6.2014).

17 Ergebnisse der Abstimmung im Sejm v. 25.1.2013 in Gazeta Wyborcza v. 25.1.2013, http://wyb orcza.pl/1,76842,13296531,Zwiazki_partnerskie_Jak_glosowali_poslowie_.html\#TRrelSST (25.6.2014) und in deutscher Sprache unter www.queer.de/detail.php?article_id=18413 (25.6.2014). 
schaftsgesetz (LPartG) ${ }^{18}$ dieser Form der Offenlegung: Hier finden wir ein Dickicht von Verweisungen auf geltende Eheregelungen, das letztlich verschleiert, welche Eheregelungen gewolltermaßen nicht entsprechend anwendbar sein sollen - und auf welche zu verweisen man geflissentlich vergessen hat.

Unter diesen Umständen kann man keineswegs von einer übereinstimmenden europäischen Rechtspraxis, sondern allenfalls von einem starken Trend in Richtung auf die Verförmlichung der Lebenspartnerschaften durch Eintragung sprechen, dem eine schwächere Tendenz zur Öffnung der Ehe für gleichgeschlechtliche Paare zur Seite tritt. Zwar haben nur relativ wenige Staaten Europas (Bulgarien, Lettland, Litauen, Polen, Ungarn) die Ehe ausdrücklich in der Verfassung auf eine Verbindung zwischen Mann und Frau beschränkt. Es gibt aber andererseits Versuche, die drohende Öffnung der Ehe für Gleichgeschlechtliche gezielt, möglichst mithilfe des Verfassungsrechts zu unterbinden. ${ }^{19}$

Inspirierend war insoweit der U.S.-amerikanische Defense of Marriage Act (DOMA 2013), der allerdings am U.S. Supreme Court scheiterte. ${ }^{20}$ Im Ergebnis umgekehrt verlief die Entwicklung in Australien, wo der High Court of Australia den Marriage Equality (Same Sex) Act 2013 eines Regionalgesetzgebers (Australian Capital Territory) zur Öffnung der Ehe für ungültig erklärte, weil er mit geltendem Bundesrecht (Marriage Act) unvereinbar war. Der High Court stellte zwar einerseits fest, dass das Ehegesetz des Bundes nur die Eheschließung zwischen Mann und Frau erlaube, andererseits aber auch, dass die Formulierung ,marriage“ in Section 51(xxi) der australischen Verfassung sich auf eine „consensual union formed between natural persons in accordance with legally prescribed requirements" beziehe und daher grundsätzlich auch gleichgeschlechtliche Paare einbeziehe; ${ }^{21}$ es kommt also letztlich auf den Willen des Bundesgesetzgebers an, während die Verfassung sich offen in alle Richtungen zeigt. Die letzteren Beispiele zeigen, dass die Kontroverse um das Wesen der Ehe auch bundesstaatliches Konfliktpotential birgt und „Ehe“ keinesfalls in allen Staaten per se als Verbindung zwischen Mann und Frau verstanden wird.

18 Lebenspartnerschaftsgesetz v. 16.2.2001, BGBl. 2001 I, 266; zuletzt geändert durch Art. 8 des Gesetzes v. 7.5.2013, BGB1. 2013 I, 1122.

19 Zum erfolgreichen Referendum in Kroatien betreffend die Aufnahme einer Definition der Ehe als „Lebensgemeinschaft von Frau und Mann“ in die Verfassung: FAZ Nr. 281 v. 3.12.2013, S. 6 - Ehe-Referendum in Kroatien spaltet die Gesellschaft. In diesem Zusammenhang soll die EU-Kommission eine Kommentierung des Referendums unter Hinweis auf die Zuständigkeit der Mitgliedstaaten für „Familienrecht und Definitionen der Ehe“ abgelehnt haben. Siehe FAZ Nr. 279 v. 30.11.2013, S. 6 - Eine Frage der Definition. Kroaten stimmen über Ehe ab.

20 U.S. Supreme Court, 570 U.S. [...] (2013), Urt. v. 26.6.2013, www.supremecourt.gov/opinions/ 12pdf/12-307_6j37.pdf (25.6.2014). Siehe auch noch unter E.

21 High Court of Australia, Urt. v. 12.12.2013, [2013] HCA 55 - The Commonwealth of Australia $v$ The Australian Capital Territory. Bis zur Ungültigerklärung sollen bereits 27 gleichgeschlechtliche Ehen im Hauptstadtgebiet geschlossen worden sein. Siehe FAZ Nr. 290 v. 13.12.2013, S. 6 - Oberstes Gericht kippt „Homo-Ehe“. 


\section{B. Rechtliche Grundlagen}

\section{Kompetenzverteilung zwischen EU und Mitgliedstaaten}

\section{Kompetenz der EU zur Bekämpfung von Diskriminierung}

Die Union besitzt die Kompetenz, Regelungen gegen Diskriminierungen aufgrund der sexuellen Orientierung zu treffen. Gemäß Art. 19 Abs. 1 EUV kann der Rat einstimmig im besonderen Gesetzgebungsverfahren ,geeignete Vorkehrungen treffen”, um solche Diskriminierungen ,zu bekämpfen“. Damit hat die EU die Möglichkeit, Sekundärrecht zu erlassen, das zwar dem Subsidiaritätsprinzip (Art. 5 Abs. 3 EUV) entsprechen muss, die Mitgliedstaaten aber durchaus zu wirksamem aktiven Handeln verpflichten darf. Gemäß Art. 19 Abs. 2 EUV können Parlament und Rat im ordentlichen Gesetzgebungsverfahren „,unter Ausschluß jeder Harmonisierung“ zudem „,die Grundprinzipien für Fördermaßnahmen der Union“ zur Unterstützung von Maßnahmen der Mitgliedstaaten festlegen.

\section{Kompetenz der Mitgliedstaaten zur Regelung des Familienstands und davon abhängiger Leistungen}

Nach Art. 7 AEUV, dem Prinzip der begrenzten Einzelermächtigung, hat die Union nur die Befugnisse, die ihr das Europarecht zuweist. Da sie unstrittig keine Kompetenz für die Regelung des Familienstandes besitzt, verbleibt diese ausschließlich bei den Mitgliedstaaten. Das klingt einfach, erweist sich aber in den Details als anspruchsvoll. So lesen wir im Erwägungsgrund 22 der Richtlinie 2000/78/EG zur Festlegung eines allgemeinen Rahmens für die Verwirklichung der Gleichbehandlung in Beschäftigung und Beruf: ${ }^{22}$

„Diese Richtlinie lässt die einzelstaatlichen Rechtsvorschriften über den Familienstand und davon abhängige Leistungen unberührt.“

Das heißt, dass die Mitgliedstaaten darüber entscheiden, ob gleichgeschlechtliche Partner eine Ehe schließen können (so z.B. in den Niederlanden), mit einer Sonderform wie der amtlich ,eingetragenen Lebenspartnerschaft” vorlieb nehmen müssen (so z.B. in Deutschland) oder auch nichts dergleichen tun können (so z.B. in Polen). Fehlt es wie beispielsweise in Polen an jeglicher „vertypten“ Form der gleichgeschlechtlichen Lebenspartnerschaft, können die Betroffenen ihre Beziehungen nur individuell durch Notarvertrag regeln. Neben dem Familienstand verweist die Formulierung zudem alle „davon abhängige Leistungen“ in die ausschließliche Regelungskompetenz der Mitgliedstaaten. Diese regeln also auch, ob und welche Leistungen im Hinblick auf das Bestehen einer Ehe oder Partnerschaft gewährt werden.

Könnte man nun argumentieren, ein bestimmter Mitgliedstaat gewährt in Abhängigkeit von der Ehe einen Familienzuschlag, der infolge seiner gezielten Anbindung an die „Ehe“ nur verheirateten, nicht aber verpartnerten Beamten und Beamtinnen zugute kommen soll? So kann es nicht gehen. Denn dann könnten die Mitgliedstaaten das Verbot der Diskrimi- 
nierung aufgrund sexueller Orientierung weitestgehend unterlaufen, indem sie Leistungen aller Art von der Eheschließung abhängig machten, die Ehe aber nur für Personen verschiedenen Geschlechts öffnen. Die ausschließliche Kompetenz der Mitgliedstaaten über die Regelung des Familienstandes und davon abhängiger Leistungen muss deshalb so interpretiert werden, dass sie mit dem europarechtlichen Verbot der Diskriminierung harmoniert. Diese Überlegung führt unvermeidlich auf das klassische „Wenn-dann-Schema“: Ein Mitgliedstaat muss verheirateten Beamten keine Familienzulage gewähren; wenn er es aber tut, dann darf er nicht aufgrund der sexuellen Orientierung diskriminieren. ${ }^{23}$

\section{Der Grundsatz der Einheitlichkeit der persönlichen Rechtsstellung}

In Personenstandsfragen erkennt der EuGH den sogenannten „Grundsatz der Einheitlichkeit der persönlichen Rechtsstellung“ an. ${ }^{24}$ Danach haben Angehörige eines Mitgliedstaats Anspruch auf Achtung ihres Personenstandes im gesamten Gebiet der EU. So vermeidet der Grundsatz der Einheitlichkeit der persönlichen Rechtsstellung Friktionen, die sich gerade daraus ergeben, dass die Unionsangehörigen einerseits ohne Nachteile von der Personenfreizügigkeit im gemeinsamen Binnenmarkt Gebrauch machen und sich dazu in andere Mitgliedstaaten begeben sollen, andererseits aber diese die persönlichen Rechtsstellungen kraft ihrer ausschließlichen Kompetenz für Familienstandsfragen sehr unterschiedlich regeln können. Gäbe es den europarechtlichen Grundsatz der Einheitlichkeit der persönlichen Rechtsstellung nicht, wäre es für Unionsangehörige sehr viel schwieriger, unberechenbarer und unattraktiver, von ihren Freizügigkeitsrechten Gebrauch zu machen. Der Grundsatz stellt deshalb eine geradezu notwendige Ergänzung zur ausschließlichen Zuständigkeit der Mitgliedstaaten in Personenstandsfragen dar. Er erinnert entfernt an das sogenannte Herkunftsland- oder Vertrauensprinzip, ${ }^{25}$ das die Geltung grundlegender Regelungen im Herkunftsstaat - zum Beispiel über die Verkehrsfähigkeit einer Ware - auf das gesamte Unionsgebiet ausdehnt und mit europaweiter Geltung versieht, aber gerade nicht uneingeschränkt gilt. ${ }^{26}$ Könnte man diesen Grundsatz auf personenstandsbegründende Akte erstrecken, würde hier der in einem EU-Staat geschlossene Ehebund ,verkehrsfähig“ oder besser „,vertrauenswürdig“ werden.

Die Probleme stecken allerdings im Detail. Denn über die Reichweite des Grundsatzes der Einheitlichkeit der persönlichen Rechtsstellung bestehen unterschiedliche Auffassungen. Welche Konsequenzen hat es genau, wenn ein gleichgeschlechtliches Paar zum Bei-

23 Zur Umsetzung dieses Ansatzes durch den EuGH noch unter C.I.1.b).

24 Siehe etwa EuGH, verb. Rs. C-122/99 P und C-125/99 P, D. und Schweden gegen Dänemark und Niederlande, Slg. 2001, I-4319, Rdnrn. 24 f., 42 ff.; sowie unter C.I.1.a).

25 Siehe zum „Herkunftsland- oder Vertrauensprinzip“ etwa Oppermann/Classen/Nettesheim, Europarecht, 6. Aufl. 2014, § 22, Rdnr. 31. Siehe auch die zahlreichen Nachweise bei Haucap/Kühling, Systemwettbewerb durch das Herkunftslandprinzip: Ein Beitrag zur Stärkung der Wachstums- und Wettbewerbsfähigkeit in der EU?, Ordnungspolitische Perspektiven Nr. 50, September 2013.

26 Ausgangspunkt für die Anerkennung des Prinzips war die Überlegung, dass der Kontakt einer Importware zu mehreren mitgliedstaatlichen Rechtsordnungen zu Mehrbelastungen führen kann, welche die Entwicklung des Binnenmarkts bremsen. Vgl. Kingreen, in: Calliess/Ruffert (Hrsg.), EUV/AEUV, 4. Aufl. 2011, Art. 34-36 AEUV, Rdnr. 149. 
spiel in Spanien die Ehe geschlossen hat und sich dann in Deutschland zum Zwecke der Aufnahme einer Beschäftigung niederlässt? Müssen auf diese gleichgeschlechtliche Ehe nach spanischem Recht alle in Deutschland für die Ehe vorgesehenen Regelungen und Vergünstigungen angewendet werden? Muss Polen die in den Niederlanden geschlossenen gleichgeschlechtlichen Ehen wie traditionelle Ehen behandeln, obwohl in diesem Land bislang noch nicht einmal die eingetragene Partnerschaft politisch durchgesetzt werden konnte? Würde man den Grundsatz der Einheitlichkeit der persönlichen Rechtsstellung hier als eine Art Herkunftslandprinzip verstehen und die betreffenden Paare in jeder Beziehung wie verheiratete behandeln, könnte die Entscheidung des deutschen oder polnischen Gesetzgebers, die Ehe noch nicht für gleichgeschlechtliche Verbindungen zu öffnen, leicht durch Eheschließung im Ausland unterlaufen werden. Wohl deshalb neigt der EuGH auch dazu, den Grundsatz der Einheitlichkeit der persönlichen Rechtsstellung sehr stark einzuschränken, nämlich auf ein Verbot, Entscheidungen zu treffen, die sich auf die personenstandsrechtliche Lage, die ein anderer Mitgliedstaat geschaffen hat, rechtsgestaltend auswirken können. ${ }^{27}$ Das bloße Vorenthalten von Leistungen, die ein Staat nur für klassische Ehepaare bereithält, verändert diese Lage jedenfalls nicht in ihrer formalen Dimension. $\mathrm{Ob}$ diese Interpretation letztlich trägt, ist eine der hier zu klärenden Fragen.

\section{Das Verbot der Diskriminierung aufgrund der sexuellen Orientierung}

\section{Die Richtlinie zur Festlegung eines allgemeinen Rahmens für die Verwirklichung der Gleichbehandlung in Beschäftigung und Beruf}

Auf der Grundlage des erwähnten Art. 19 Abs. 1 EUV (früher Art. 13 EGV) hat der Rat die Richtlinie 2000/78/EG zur Festlegung eines allgemeinen Rahmens für die Verwirklichung der Gleichbehandlung in Beschäftigung und Beruf ${ }^{28}$ verabschiedet. Sie ist für Deutschland, soweit es das Merkmal der sexuellen Orientierung betrifft, regulär, nämlich mit Ablauf der Umsetzungsfrist am 2. Dezember 2000 (Art. 20), wirksam geworden. ${ }^{29}$

Die Richtlinie bezweckt die Schaffung eines ,allgemeinen Rahmens“ zur Bekämpfung von Diskriminierung unter anderen aus Gründen der sexuellen Orientierung in den Bereichen „Beschäftigung und Beruf“ (Art. 1 und 3). Es darf, wie Art. 2 klarstellt, „,keine unmittelbare oder mittelbare Diskriminierung [...] geben“. Ausdrücklich ausgeschlossen wird die Anwendung der Richtlinie auf Leistungen der Sozialversicherung und Sozialschutzes, sofern diese nicht unter bestimmten Umständen dem Arbeitsentgelt im Sinne von Art. 157 AEUV (ex-Art. 141 EGV) gleichgestellt sind. Diskriminierungen im Sozialversicherungsbereich, etwa bei Rentenansprüchen, unterfallen also nur ausnahmsweise der Richtlinie, die auf „Beschäftigung und Beruf“ bezogen ist. Ebenfalls nicht von der Richtlinie erfasst sind staatliche Leistungen, die dem Zugang zur Beschäftigung oder dem Erhalt derselben

27 Siehe EuGH, verb. Rs. C-122/99 P und C-125/99 P, D. und Schweden gegen Dänemark und Niederlande, Slg. 2001, I-4319, Rdnr. 43; sowie noch unter C.I.1.a).

28 Siehe Fn. 22.

29 Anderes gilt für das Merkmal des Geschlechts. Hier hat Deutschland von der Möglichkeit der Verlängerung der Umsetzungsfrist gemäß Art. 18 Abs. 2 RL 2000/78/EG Gebrauch gemacht. Siehe EuGH, Rs. C-147/08, Römer, Slg. 2011, I-3591, Rdnr. 58. 
dienen (Erwägungsgrund 13). Die Richtlinie konzentriert sich auf im weiteren Sinne entgeltbezogene Diskriminierungen, die mit dem von der Arbeitgeberseite gezahlten Lohn und nicht mit speziellen Arbeitsfördermaßnahmen des Staates zusammenhängen.

Einzelpersonen können sich unmittelbar vor nationalen Gerichten und Behörden auf die Richtlinie berufen, sofern diese nach Ablauf der Umsetzungsfrist entweder gar nicht, nicht vollständig oder nicht richtig umgesetzt wurde und die betreffende Vorschrift der Richtlinie sich für eine unmittelbare Anwendbarkeit eignet, wie insbesondere das aus Art. 2 der Richtlinie folgende Verbot der Diskriminierung oder die Maßstäbe für die Feststellung einer erlaubten bzw. unerlaubten Differenzierung. Die unmittelbare Anwendbarkeit der Richtlinie hat der EuGH inzwischen auch speziell mit Bezug auf Diskriminierungen aufgrund der sexuellen Orientierung bestätigt. ${ }^{30}$ Da Deutschland die Richtlinie durch das Allgemeine Gleichbehandlungsgesetz (AGG) grundsätzlich umgesetzt hat, ${ }^{31}$ kommt eine direkte Anwendung nur in Bezug auf einzelne Umsetzungsdefizite in Betracht. Allerdings muss zunächst das AGG so ausgelegt werden, dass es dem Europarecht entspricht. ${ }^{32}$

\section{Das Verbot der Diskriminierung aufgrund sexueller Orientierung als Grundrecht der EU (Art. 6 EUV; Art. 21 GrCh; Art. 14 in Verbindung mit Art. 8 EMRK)}

Was gilt, wenn eine Diskriminierung aufgrund der sexuellen Orientierung infrage kommt, die Angelegenheit jedoch nicht in den Bereich von „Beschäftigung und Beruf“ im Sinne der Richtlinie 2000/78/EG fällt? Auch dann verbietet das Recht der Europäischen Union die Diskriminierung, soweit die Angelegenheit aus anderen Gründen im Anwendungsbereich des Europarechts liegt. Unter diesen Umständen griff bereits früher ein allgemeines Verbot der Diskriminierung im Sinne eines ungeschriebenen europäischen Grundrechts, das der EuGH ursprünglich im Zusammenhang mit Diskriminierungen aufgrund des Geschlechts entwickelt hatte. ${ }^{33}$ Inzwischen hat diese Rechtsprechung auch Niederschlag in den europäischen Verträgen gefunden, wobei der einschlägige Art. 6 EUV in erster Linie auf die Charta der Grundrechte, in zweiter Linie auf die EMRK und in dritter Linie auf die Verfassungsüberlieferungen der Mitgliedstaaten verweist. Artikel $21 \mathrm{GrCh}$ enthält ein ausdrückliches und spezielles Verbot der Diskriminierung aufgrund der sexuellen Orientierung, das vermittels Art. 6 Abs. 3 EUV als allgemeiner Grundsatz des Europarechts mit Primärrechtsrang gilt.

Auch aus der EMRK, auf die Art. 6 EUV ebenfalls verweist, wird ein solches Verbot abgeleitet: Hier gilt die sexuelle Orientierung als „,sonstiger Status“, aufgrund dessen gemäß Art. 14 EMRK nicht diskriminiert werden darf. ${ }^{34}$ Darüber hinaus ist das homosexuelle

30 Ibid., Rdnr. 56.

31 Gesetz zur Umsetzung europäischer Richtlinien zur Verwirklichung des Grundsatzes der Gleichbehandlung v. 14.8.2006, BGBl. 2006 I, 1897. Siehe auch Rust, in: Rust/Falke (Hrsg.), AGG, 2007, Einleitung, Rdnrn. 1, 273; § 1, Rdnr. 2.

32 Dazu auch Bruns, Die Maruko-Entscheidung im Spannungsfeld zwischen europäischer und nationaler Auslegung, NJW 2008, S. 1931.

33 Grundlegend EuGH, Rs. 149/77, Defrenne III, Slg. 1978, 1365, 1379.

34 Siehe Peters/König, Das Diskriminierungsverbot, in: Dörr/Grote/Marauhn (Hrsg.), EMRK/GG, 2. Aufl. 2013, Kap. 21, Rdnr. 198 ff.; Wiemann, Die Rechtsprechung des EGMR zu sexueller Orientierung, EuGRZ 2010, S. 408 ff. 
Zusammenleben als solches durch Art. 8 EMRK (Recht auf Privat- und Familienleben) geschützt, wobei die Fälle einesteils anhand von Art. 8 EMRK, anderenteils anhand von Art. 14 in Verbindung mit Art. 8 EMRK und eher selten auf beiden Grundlagen zugleich geprüft werden. ${ }^{35}$ Wie der EGMR im Fall Karner gegen Österreich klargestellt hat, sind Differenzierungen aufgrund der sexuellen Orientierung nur aus „,besonders schwerwiegenden Gründen“ (,particularly serious reasons“) zu rechtfertigen. ${ }^{36}$ Besonderheiten wie diese bei der Kontrolldichte zeigen, warum der Rückgriff auf die EMRK ungeachtet einer ausdrücklichen Regelung in Art. $21 \mathrm{GrCh}$ seine Bedeutung behält. Das gilt umso mehr, als Art. 52 Abs. 3 GrCh einen Gleichklang zwischen beiden Instrumenten vorsieht.

Erwähnenswert ist schließlich, dass die Union gemäß Art. 10 AEUV in ihrer ganzen Politik auf die Bekämpfung von Diskriminierungen, unter anderen solcher aufgrund sexueller Orientierung, verpflichtet ist. Dem entsprechend sind ihre Maßnahmen und Regelungen nicht nur im Lichte des grundrechtlichen Verbots der Diskriminierung (Art. 6 EUV, GrCh, EMRK), sondern auch im Lichte des Auftrags aus Art. 10 AEUV zu interpretieren.

\section{Recht auf Ehe aus Art. 6 EUV in Verbindung mit Art. 9 GrCh, Art. 12 EMRK?}

Auf den Kern der Thematik zielt die weitergehende Frage, ob das europäische Recht Homosexuellen nicht schon heute das Recht auf Eheschließung garantiert. Neue Maßstäbe der Bewertung hat insoweit Art. 9 GrCh (Eheschließungsfreiheit) gesetzt:

„Das Recht, eine Ehe einzugehen, und das Recht, eine Familie zu gründen, werden nach den einzelstaatlichen Gesetzen gewährleistet, welche die Ausübung dieser Rechte regeln.“

Wie die quasi-offizielle Kommentierung zu dieser Bestimmung erläutert, hat man hier im Unterschied zu allen anderen internationalen Texten zur Eheschließungsfreiheit von jedem Hinweis auf Mann und Frau abgesehen, um die Norm auch für nicht-traditionelle Ehen zu öffnen - allerdings nur, sofern die jeweilige staatliche Gesetzgebung dies so vorsieht. ${ }^{37}$ Der Kommentar interpretiert dies so, dass Art. 9 GrCh der Anerkennung gleichgeschlechtlicher Beziehungen im Kontext der Ehe nicht entgegensteht, sie aber auch nicht einfordert. ${ }^{38}$ Diese Entwicklung hat wiederum auf Art. 12 EMRK (Eheschließungsfreiheit) zurückgewirkt, in dem auch dort die generelle Beschränkung der Norm auf Verbindungen zwischen Mann und Frau aufgegeben wurde, obwohl auch hier die konkrete Ermöglichung gleichgeschlechtlicher Ehen den einzelnen Vertragsstaaten überlassen bleibt. ${ }^{39}$ Dieses Grundmuster der Öffnung der Eheschließungsfreiheit bei gleichzeitiger Betonung einzelstaatlicher

35 Siehe Fn. 79.

36 EGMR, Nr. 40016/98, Karner, Rep. 2003-IX, Rdnr. 37.

37 Eriksson, in: EU Network of Independent Experts, Commentary of the Charter of Fundamental Rights of the European Union, Juni 2006, S. 98.

38 Ibid., S. 102. Die deutsche Kommentarliteratur gibt dies im Wesentlichen unkommentiert wieder. Siehe etwa Folz, in: Vedder/Heintschel v. Heinegg (Hrsg.), Europäisches Unionsrecht, 2012, Art. 9 GR-Charta, Rdnr. 3; Schorkopf, in: Ehlers (Hrsg.), Europäische Grundrechte und Grundfreiheiten, 3. Aufl. 2009, §16.1, Rdnr. $53 \mathrm{f}$. Siehe nun auch EGMR, Nr. 30141/04, Urt. v. 24.6.2010, Schalk und Kopf, Rdnr. 25.

39 Eingehend dazu unter C.II. 
Gestaltungsmacht prägt das heutige Verständnis der menschenrechtlichen Bestimmungen im fraglichen Bereich.

\section{Anerkennung des Partnerschafts- oder Ehestatus in anderen EU-Ländern?}

\section{Der Ehebegriff des europäischen Verfahrensrechts}

Soweit es die internationale Zuständigkeit in Ehesachen und die Anerkennung von Entscheidungen auf diesem Gebiet betrifft, sind spezielle Regelungen zum Ehestatus in der Verordnung (EG) Nr. 2201/2003 (EheVO II bzw. „Brüssel IIa“) ${ }^{40}$ enthalten. Unterhaltsfragen im Zusammenhang mit der Ehe sind dagegen in der Verordnung (EG) Nr. 44/2001 (EuGVO, „Brüssel I“) ${ }^{41}$ geregelt. Insoweit stellt sich die interessante Frage, ob gleichgeschlechtliche Ehen, wie es sie in einigen Mitgliedstaaten schon gibt, unter den Ehebegriff dieser Verordnungen fallen. Das wird nicht nur von Teilen der Literatur, ${ }^{42}$ sondern offenbar auch von den Niederlanden so vertreten; ${ }^{43}$ während vereinzelte Rechtsprechung sich eher ablehnend äußert(e). ${ }^{44}$ Eine Klärung durch den EuGH steht insoweit noch aus.

Die Konsequenz einer Anerkennung der gleichgeschlechtlichen Ehe als „Ehe“ im Sinne der EheVO wäre, dass aufgrund der Aufenthaltszuständigkeit für Ehescheidungen (Art. 3 EheVO) unter Umständen die Gerichte eines Staates über die Auflösung einer „Homo-Ehe“ entscheiden müssten, deren Recht eine solche Ehe gar nicht kennt. Der Fall tritt zum Beispiel ein, wenn ein gleichgeschlechtliches niederländisches Ehepaar seinen Wohnsitz nach Deutschland verlegt, so dass die Scheidung wegen der Aufenthaltszuständigkeit (Art. 3 EheVO) nach deutschem Recht vorzunehmen ist. Während die Einen diese Konsequenz als nicht ungewöhnlich für das IPR erachten, ${ }^{45}$ sehen die Anderen hier ein Transpositionsoder Substitutionsproblem: Da das anwendbare deutsche Recht auf diesen Fall nicht eingestellt sei, seien ersatzweise die Regelungen über die Auflösung eingetragener Partnerschaften heranzuziehen. ${ }^{46}$ Man könnte natürlich auch argumentieren: Es handelt sich mit Blick auf die Schließung nach niederländischem Recht um eine „Ehe“, also muss diese

40 VO (EG) Nr. 2201/2003 des Rates über die Zuständigkeit und die Anerkennung und Vollstreckung von Entscheidungen in Ehesachen und in Verfahren betreffend die elterliche Verantwortung und zur Aufhebung der VO (EG) Nr. 1347/2000 v. 27.11.2003, ABl. L 338 v. 23.12.2003, S. 1 ff. (im Folgenden: EheVO).

41 VO (EG) Nr. 44/2001 des Rates v. 22.12.2000 über die gerichtliche Zuständigkeit und die Anerkennung und Vollstreckung von Entscheidungen in Zivil- und Handelssachen, ABl. L 12 v. 16.1.2001, S. 1 .

42 Winkler v. Mohrenfels, (Fn. 14), S. 539 mit Nachweisen zur Gegenmeinung. Siehe aber auch Streinz, EUV/AEUV, 2. Aufl. 2012, Art. 9 GrCh, Rdnr. 4 f., der die Einbeziehung gleichgeschlechtlicher Ehen in den unionsrechtlichen Ehebegriff nur mit Blick auf ältere Praxis ablehnt, im Übrigen aber jedenfalls keine Verpflichtung der Mitgliedstaaten zur Einbeziehung sieht.

43 Nachweise bei Winkler v. Mohrenfels, (Fn. 14), S. 538 f. mit Fn. 73.

44 Siehe zu inzwischen überholtem Sekundärrecht noch VG Karlsruhe, Urt. v. 9.9.2004, BeckRS 2004, 24618: Ein Nicht-Unions-Bürger, der eine gleichgeschlechtliche Ehe mit einem nach Art. 39 EG-Vertrag (heute: Art. 45 AEUV) freizügigkeitsberechtigten Arbeitnehmer geschlossen hat, ist kein „Ehegatte“ i.S.v. Art. 10 Abs. 1 lit. a der VO (EWG) Nr. 1512/68.

45 Winkler v. Mohrenfels, (Fn. 14), S. 539.

46 Coester, in: Münchener Kommentar zum BGB, Bd. 10: IPR, 5. Aufl. 2010, Art. 13 EGBGB, Rdnr. 52. 
auch in Deutschland als „Ehe“ behandelt werden. Damit würde man allerdings die gleichgeschlechtliche Ehe durch die Hintertür in die anderen Mitgliedstaaten importieren. ${ }^{47}$

Die deutsche Rechtslage verdeutlicht beispielhaft, wie schlecht das geltende Kollisionsrecht seine Aufgabe erfüllt. Hier wurde nämlich nur der Fall einer in anderen EU-Mitgliedstaaten begründeten eingetragenen Partnerschaft, nicht jedoch der Fall einer gleichgeschlechtlichen Ehe bedacht - obwohl der für Partnerschaften einschlägige Art. 17b $\mathrm{EGBGB}^{48}$ ca. zwei Monate nach dem niederländischen Gesetz zur Einführung der gleichgeschlechtlichen Ehe vom 21. Dezember 2000 verabschiedet wurde. ${ }^{49}$ Wendet man auf eine solche in den Niederlanden geschlossene Ehe Art. 17b EGBGB an, müssen die Ehegatten im Geltungsbereich deutschen Rechts mit der Rechtsstellung von eingetragenen Lebenspartnern, also einer Stellung minderen Rechts, vorlieb nehmen. Beurteilt man sie dagegen nach dem für klassische Ehen vorgesehenen Art. 13 EGBGB, richten sich die materiellen Voraussetzungen der Eheschließung nach dem jeweiligen Heimatstaat (Personalstatut), so dass es auf die Staatsangehörigkeit der beiden Verlobten ankommt: Handelt es sich zum Beispiel um ein deutsch-niederländisches Paar, kann dieses zwar in den Niederlanden nach dortigem Recht die Ehe schließen; Art. 13 EGBGB unterwirft den deutschen Teil aber deutschem Recht, mit der Folge, dass die geschlossene Ehe (nur) für den deutschen Teil unwirksam sein soll. ${ }^{50}$ In diesem Falle klafften also deutsches und niederländisches Recht auseinander und könne im deutsch-niederländischen Verhältnis eine „hinkende Ehe” entstehen. ${ }^{51}$

Dass Art. 13 EGBGB sich überhaupt in dieser Weise auf bereits im Ausland geschlossene Ehen auswirken kann, überrascht, da sich die Vorschrift ausdrücklich nur auf die „Voraussetzungen“ der Eheschließung und dem entsprechend die „Verlobten“ bezieht. ${ }^{52}$ Gleichwohl haben sich Rechtsprechung und herrschende Lehre mit der Begründung, die Betroffenen vor dem Unheil einer hinkenden Ehe (als Folge der Anwendung von Art. 13 EGBGB) bewahren zu wollen, der sogenannten „funktionalen“ Betrachtungsweise angeschlossen. Danach komme nur Art. 17b EGBGB in Betracht; denn maßgeblich sei nicht die Bezeichnung „Ehe“, sondern die Art. 17b EGBGB innewohnende Funktion, jede rechtsförmliche gleichgeschlechtliche Verbindung in Deutschland als eingetragene Lebenspartnerschaft zu

47 Siehe allerdings VO (EU) Nr. 1259/2010 des Rates v. 20.12.2010 zur Durchführung einer Verstärkten Zusammenarbeit im Bereich des auf die Ehescheidung und Trennung ohne Auflösung des Ehebandes anzuwendenden Rechts, AB1. L 343 v. 29.12.2010, S. 10. Diese VO, mit der eine Reihe von Staaten einschließlich Deutschland eine verstärkte Zusammenarbeit begründeten, soll einen gemeinsamen Rechtsrahmen für Ehescheidungen begründen, geht allerdings nicht explizit auf die Problematik der gleichgeschlechtlichen Ehe ein. In Erwägungsgrund 24 ist lediglich von „bestimmten Situationen“ die Rede, ,in denen das anzuwendende Recht eine Ehescheidung nicht zulässt oder einem der Ehegatten aufgrund seiner Geschlechtszugehörigkeit keinen gleichberechtigten Zugang zu einem Scheidungs- oder Trennungsverfahren gewährt“.

48 Art. 1 Gesetz zur Beendigung der Diskriminierung gleichgeschlechtlicher Lebenspartnerschaften v. 16.2.2001, BGB1. 2001 I, 266. Siehe dazu BT-Drs. 14/3751, S. 60.

49 Siehe Winkler v. Mohrenfels, (Fn. 14), S. 530.

50 OLG München, Beschl. v. 6.7.2011, BeckRS 2011, 18723.

51 Siehe ibid.; Winkler v. Mohrenfels, (Fn. 14), S. 536 m.w.N.

52 Siehe auch Mörsdorf-Schulte, in: Bamberger/Roth, Beck'scher Online-Kommentar BGB, Art. 13 EGBGB, Rdnr. 20: Art. 17b sei analog anzuwenden, da sein präzise gefasster Wortlaut keine unmittelbare Anwendung zulasse. 
erfassen; somit könnten verehelichte gleichgeschlechtliche Paare in Deutschland nur die Eintragung in das Lebenspartnerschaftsregister erreichen („Grundsatz des schwächeren Rechts"), dies aber selbst dann, wenn infolge der ausländischen Eheschließung die speziellen Eintragungsvoraussetzungen für eine eingetragene Partnerschaft nicht erfüllt seien. ${ }^{53}$

Ähnlich schwierig gestaltet sich die Lage für unser deutsch-niederländisches Paar aber auch im Bereich der Ehewirkungen, da Art. 14 EGBGB diese grundsätzlich am Recht des Staates des gewöhnlichen Aufenthalts orientiert, so dass jedenfalls bei einer Wohnsitznahme in Deutschland nicht die für Ehen geltenden Regelungen anwendbar wären. Interessant ist insoweit der Vergleich mit polygamen Ehen, die bei Beteiligung einer Person deutscher Staatsangehörigkeit zwar aufgehoben werden „,kann“ ( $\S ~ 1306,1314$ Abs. 1 BGB; Art. 13 EGBGB), aber doch grundsätzlich respektierbar ist. ${ }^{54}$ Warum die im Ausland entstandene Mehrehe besser gestellt sein soll als die gleichgeschlechtliche Ehe, leuchtet nicht ein.

Für den Bereich des IPR lässt sich nach allem festhalten, dass die Entscheidung eines EU-Staates, die gleichgeschlechtliche Ehe anzuerkennen, von anderen Mitgliedstaaten, die dieses Institut nicht kennen, kollisionsrechtlich nicht akzeptiert werden muss und zumeist auch nicht akzeptiert wird. Das deutsche Kollisionsrecht ist von Notlösungen geprägt (,funktionale Betrachtungsweise“) und nicht frei von Wertungswidersprüchen (höhere Akzeptanz für polygame als für gleichgeschlechtliche Ehen).

\section{Gleichgeschlechtliche Lebens- oder Ehepartner als „Familienangehörige“}

Bei der Frage, inwieweit gleichgeschlechtliche Lebens- oder Ehepartner als „Familienangehörige" gelten, zeigt sich das Europarecht zurückhaltend. Das gilt insbesondere für Regelungen, welche die Einreise in ein anderes Mitgliedsland und den Aufenthalt dort betreffen.

\section{a) Regelungsstrategien der Union: Aufenthaltsrichtlinie und Familienzusammenführungsrichtlinie im Lichte grundrechtlicher Grenzen}

Die Aufenthaltsrichtlinie 2004/38/EG ${ }^{55}$ regelt, inwieweit Unionsangehörige ihre Familienangehörigen ungeachtet der jeweiligen Staatsangehörigkeit in ein anderes Mitgliedsland mitnehmen können und welche Rechte sie dort haben.

53 OLG München, Beschl. v. 6.7.2011, BeckRS 2011, 18723 (gleichgeschlechtliche Ehe zwischen Deutschem und Spanier nach spanischem Recht); OLG Zweibrücken, Beschl. v. 21.3.2011, BeckRS 2011, 14240 (gleichgeschlechtliche Ehe nach kalifornischem Recht); OLG Köln, Beschl. v. 5.7.2010, BeckRS 2010, 22293 (gleichgeschlechtliche Ehe zweier Deutscher in „M.“).

54 Siehe Winkler v. Mohrenfels, (Fn. 14), S. 537.

55 RL 2004/38/EG des Europäischen Parlaments und des Rates v. 29.4.2004 über das Recht der Unionsbürger und ihrer Familienangehörigen, sich im Hoheitsgebiet der Mitgliedstaaten frei zu bewegen und aufzuhalten, zur Änderung der VO (EWG) Nr. 1612/68 und zur Aufhebung der RL 64/221/EWG et al., ABl.L 158 v. 30.4.2004, S. 77; berichtigte Fassung, ABl. L 229 v. 29.6.2004, S. 35 . 
In Erwägungsgrund 5 der Aufenthaltsrichtlinie heißt es:

„Das Recht aller Unionsbürger, sich im Hoheitsgebiet der Mitgliedstaaten frei zu bewegen und aufzuhalten, sollte, wenn es unter objektiven Bedingungen in Freiheit und Würde ausgeübt werden soll, auch den Familienangehörigen ungeachtet ihrer Staatsangehörigkeit gewährt werden. Für die Zwecke dieser Richtlinie sollte der Begriff des Familienangehörigen auch den eingetragenen Lebenspartner umfassen, wenn nach den Rechtsvorschriften des Aufnahmemitgliedstaats die eingetragene Partnerschaft der Ehe gleichgestellt wird.“

Diese Leitlinie führt Art. 2 Abs. 2 lit. b Aufenthaltsrichtlinie folgendermaßen aus:

„Im Sinne dieser Richtlinie bezeichnet der Ausdruck ,Familienangehöriger' [...]

a) den Ehegatten;

b) den Lebenspartner, mit dem der Unionsbürger auf der Grundlage der Rechtsvorschriften eines Mitgliedstaats eine eingetragene Partnerschaft eingegangen ist, sofern nach den Rechtsvorschriften des Aufnahmemitgliedstaats die eingetragene Partnerschaft der Ehe gleichgestellt ist und die in den einschlägigen Rechtsvorschriften des Aufnahmemitgliedstaats vorgesehenen Bedingungen erfüllt sind; $[\ldots] . “$

Die Aufenthaltsrichtlinie erläutert nicht, was sie unter „Ehegatten“ versteht, ${ }^{56}$ sondern geht nur auf die eingetragene Partnerschaft näher ein. Insoweit verweist sie ganz auf die Anforderungen des Aufnahmestaates und setzt dessen Bereitschaft zur Gleichstellung mit der Ehe voraus. Das heißt nur der gleichgestellte Partner, nicht jeder eingetragene Partner, kann danach „Familienangehöriger“ sein; die fehlende Bereitschaft eines Mitgliedstaats, gleichstellen zu wollen, wird ungeachtet einer dort bestehenden Eintragungsoption für die Partnerschaft maßgeblich. Zugleich gibt die Richtlinie als Grundsatz zu erkennen, dass ein Mitgliedstaat, der eingetragene Partnerschaften selbst nicht eingeführt und der Ehe gleichgestellt hat, auch nicht die eingetragenen Lebenspartner aus anderen Mitgliedstaaten als einreise- und aufenthaltsberechtigte Familienangehörige akzeptieren muss. Das Vertrauens- oder Äquivalenzprinzip, wonach zum Beispiel im Bereich des Warenverkehrs die Rechtsakte anderer Mitgliedstaaten innerhalb des gemeinsamen Rechtsraums als gleichwertig anerkannt werden müssen, ${ }^{57}$ wird hier also mit Nachdruck verneint.

Vergleichbares gilt für die Richtlinie zur Familienzusammenführung (2003/86/EG) ${ }^{58}$ Sie regelt, inwieweit eine Person, die in einem EU-Staat einen Aufenthaltstitel mit der begründeten Aussicht auf dauerhaften Aufenthalt besitzt, ihre drittstaatsangehörigen Familienmitglieder zu sich in die EU bzw. den jeweiligen Aufenthaltsstaat holen kann (Art. 3 Abs. 1). Sie gilt ausdrücklich nicht für die Familienangehörigen von Unionsbürgern bzw. -bürgerinnen (Art. 3 Abs. 3), da diese der Aufenthaltsrichtlinie unterfallen. Ein Fall für die Familienzusammenführungsrichtlinie ist zum Beispiel der algerische Staatsangehörige, der eine Aufenthaltserlaubnis für Frankreich besitzt und seine algerische Ehefrau und die minderjährigen Kinder zum Zwecke der Familienzusammenführung nach Frankreich nachholen will.

56 Dazu noch im Folgenden b).

57 Grundlegend EuGH, Rs. 120/78, Cassis de Dijon, Slg. 1979, 649, Leitsätze 2 und 3, Rdnrn. 8, 14. Siehe auch EuGH, Rs. C-212/97, Centros, Slg. 1999, I-1459, insbes. Rdnr. 27.

58 RL 2003/86/EG v. 22.9.2003 betreffend das Recht auf Familienzusammenführung, AB1. L 251 v. 3.10.2003, S. 12 . 
Auch hier gibt Art. 4 der Richtlinie 2003/86/EG dem drittsstaatsangehörigen Ehepartner ein Recht auf Einreise und Aufenthalt, dem Lebenspartner aber nur eine Option, die völlig in der Gestaltungsmacht des aufnehmenden Mitgliedstaats liegt:

„(1) Vorbehaltlich der in Kapitel IV ${ }^{59}$ sowie in Artikel $16^{60}$ genannten Bedingungen gestatten die Mitgliedstaaten gemäß dieser Richtlinie folgenden Familienangehörigen die Einreise und den Aufenthalt:

a) dem Ehegatten des Zusammenführenden; [...]

(3) Vorbehaltlich der in Kapitel IV genannten Bedingungen können die Mitgliedstaaten in ihren nationalen Rechtsvorschriften dem nicht ehelichen Lebenspartner, der Drittstaatsangehöriger ist und der nachweislich mit dem Zusammenführenden in einer auf Dauer angelegten Beziehung lebt, oder einem Drittstaatsangehörigen, der mit dem Zusammenführenden eine eingetragene Lebenspartnerschaft gemäß Artikel 5 Absatz $2^{61}$ führt, [...], die Einreise und den Aufenthalt gemäß dieser Richtlinie gestatten.“

Zudem stellt Erwägungsgrund 10 der Richtlinie 2003/86/EG klar, dass die Mitgliedstaaten das Entscheidungsrecht darüber behalten, ob sie die Familienzusammenführung auch auf Lebenspartner erstrecken wollen oder nicht. Selbst wenn sie das tun, müssen sie die Lebenspartner aus anderen Mitgliedsländern aber nicht als Familienangehörige im Sinne des europäischen Rechts, sondern dürfen sie auch schlechter behandeln:

„(10) Es ist Sache der Mitgliedstaaten zu entscheiden, ob sie die Familienzusammenführung von [...] nicht ehelichen Lebenspartnern oder eingetragenen Lebenspartnerschaften, sowie [...] zulassen möchten. Gestattet ein Mitgliedstaat die Zusammenführung dieser Personen, so gilt dies im Falle von Mitgliedstaaten, die das Bestehen familiärer Bindungen in den von dieser Bestimmung erfassten Fällen nicht anerkennen, unbeschadet der Möglichkeit, diesen Personen hinsichtlich des Rechts, sich in einem anderen Mitgliedstaat aufzuhalten, die Behandlung von Familienangehörigen im Sinne des einschlägigen Gemeinschaftsrechts zu versagen.“

Auf den ersten Blick müssen die Mitgliedstaaten nur Ehegatten Einreise und Aufenthalt gestatten, während eingetragene Partner nur entsprechend berechtigt werden können. Ihnen gegenüber kann auch eine Familienzusammenführung minderen Rechts entstehen, die sich ganz an den Vorstellungen des Aufnahmestaates orientiert. Allerdings dürfen die Richtlinien nicht isoliert betrachtet, sondern muss mit bedacht werden, dass das Zusammenleben mit dem Lebenspartner grundrechtlich geschützt und die ungerechtfertigte Diskriminierung verboten ist (Art. 6 EUV in Verbindung mit Art. 8, 14 EMRK). Aber nicht nur die Richtlinien selbst, sondern auch die zu ihrer Durchführung ergriffenen Maßnahmen der Mitgliedstaaten (Art. 52 Abs. 5 GrCh) sind im Lichte der EU-Grundrechte zu interpretieren. Die Freiheit der Mitgliedstaaten bei der Behandlung von gleichgeschlechtlichen Partnern aus anderen Mitgliedstaaten ist dem entsprechend grundrechtlich begrenzt; sie unterliegt mindestens den Erfordernissen der Gesetzmäßigkeit und der Verhältnismäßigkeit.

59 Vorbehalt der öffentlichen Sicherheit und Ordnung; Nachweise über Wohnraum, Krankenversicherung, Einkünfte etc.

60 Falsche Angaben, Scheinehe etc.

61 Vorlage von Dokumenten über die familiäre Bindung. 


\section{b) Begriff des „Ehegatten” im Sekundärrecht und Öffnung der Ehe in einzelnen Mitgliedstaaten}

Sowohl die Aufenthalts- als auch die Familienzusammenführungsrichtlinie treffen, wie gezeigt, eine scharfe Unterscheidung zwischen dem Begriff des „Ehegatten“, der alle Rechte des mitbegünstigten Familienangehörigen erhält und dem Lebenspartner, der letztlich dem - liberalen oder restriktiven - Recht des Aufnahmelandes unterworfen wird. Das kann für den eingetragenen Lebenspartner bedeuten, dass er in anderen EU-Ländern genauso wie jemand behandelt wird, der locker und unverbindlich mit einer anderen Person zusammenlebt. Was gilt aber für gleichgeschlechtliche Ehen, wie sie inzwischen acht EULänder kennen? Kann die brasilianische Ehepartnerin der portugiesischen EU-Bürgerin beanspruchen, dass sie mit Blick auf ihre in Portugal geschlossene Ehe in Österreich „Ehegattin“ im Sinne des EU-Sekundärrechts ist? Kann der Schwede von Deutschland verlangen, dass seinem nigerianischen Ehepartner alle Privilegien eines Familienangehörigen im Sinne des EU-Rechts gewährt werden? Als die beiden Richtlinien zur Familienzusammenführung und zum Aufenthalt 2003 und 2004 verabschiedet wurden, gab es die gleichgeschlechtliche Ehe immerhin schon in den Niederlanden und in Belgien. Dennoch haben die Richtlinien diesen Fall ganz offensichtlich nicht bedacht.

Es trifft aber auch ein sehr viel jüngerer Rechtsakt, nämlich die Richtlinie 2010/41/EU über die Gleichbehandlung von Männern und Frauen bei der Ausübung einer selbstständigen Erwerbstätigkeit, ${ }^{62}$ wieder dieselbe grundsätzliche Unterscheidung: Ehepartner werden uneingeschränkt durch die Richtlinie geschützt, Lebenspartner hingegen nur, ,soweit sie nach innerstaatlichem Recht anerkannt sind" (Art. 2 lit. b), also der Empfangsstaat sie anerkennt. Dass zum Zeitpunkt der Verabschiedung der Richtlinie schon sieben(!) EUStaaten die Ehe für gleichgeschlechtliche Paare geöffnet hatten, ${ }^{63} \mathrm{kann}$ dem europäischen Gesetzgeber schlechterdings nicht mehr entgangen sein. Wenn er den Fall dennoch nicht als eigene Kategorie anspricht, kann eigentlich nur die Kategorie „Ehepartner“ für gleichgeschlechtliche Ehen einschlägig sein. Andererseits kann dies zu dem widersinnigen Ergebnis führen, dass die Richtlinie Lebenspartner nur begünstigt, wenn der Empfangsstaat die eingetragene Partnerschaft anerkennt, er die viel weiter gehende gleichgeschlechtliche Ehe aber obligatorisch anerkennen muss. Was hier allerdings als regelungstechnischer Widerspruch daherkommt, könnte letztlich Folge eines aus Uneinigkeit entstandenen Schweigens sein: Es ist eine Sache, die eingetragene Partnerschaft eines anderen Mitgliedstaats nicht anzuerkennen, weil man dieses Rechtsinstitut selbst bewusst nicht eingeführt hat; eine andere Sache ist es jedoch, einer „Ehe“, die nach dem Recht eines anderen Mitgliedstaates genau unter dieser Bezeichnung besteht und die alle Mitgliedstaaten als Rechtsinstitut kennen, die Anerkennung als familienbegründenden Akt zu versagen. Deshalb ist es nicht aus systematischen Gründen ausgeschlossen, den Begriff des „Ehegatten“ im Sekundärrecht offen zu interpretieren, d.h. auch auf gleichgeschlechtliche Ehegatten zu erstrecken. Im Gegenteil: Dafür, dass „Ehe“ jede Ehe meint, sofern nicht bestimmte Fälle davon aus-

62 RL 2010/41/EU des Europäischen Parlaments und des Rates zur Verwirklichung des Grundsatzes der Gleichberechtigung von Männern und Frauen, die selbstständige Erwerbstätigkeit ausüben, und zur Aufhebung der RL 86/613/EWG des Rates v. 7.7.2010, ABl. L 180 v. 15.7.2010, S. 1.

63 Siehe unter A.III. 
drücklich ausgenommen sind, spricht mit dem Wortlaut aller Anschein, so dass hier die Vermutung einer umfassenden Regelung der Familienzugehörigkeit aller Ehepartner greift.

\section{Rechtspraxis}

\section{EuGH}

\section{Ausgewählte Fälle}

Die im Folgenden behandelten Fälle verdeutlichen exemplarisch, welche Bedeutung der Grundsatz der Einheitlichkeit der persönlichen Rechtsstellung hat (Fall $D$.) und wie weit das europarechtliche Diskriminierungsverbot in Bezug auf die Gleichstellung von Ehe und eingetragener Partnerschaft reicht bzw. wie dies mit der Interpretation des Begriffes „Arbeitsentgelt“" zusammenhängt (Fälle Maruko; Dittrich, Klinke und Müller).

\section{a) D. und Königreich Schweden (2001)}

Im Fall D. und Königreich Schweden $(2001)^{64}$ ging es um die Frage, ob ein Beamter der damaligen EG, der eine eingetragene Partnerschaft nach schwedischem Recht begründet hatte, in Bezug auf die Zahlung einer Haushaltszulage einem verheirateten Beamten gleichzustellen war. $D$. hatte argumentiert, dass die Verweigerung der Gleichstellung mit verheirateten Beamten den Grundsatz der Einheitlichkeit der persönlichen Rechtsstellung verletze, wonach jeder Angehörige eines EU-Mitgliedstaates Anspruch darauf habe, dass sein im Herkunftsstaat begründeter Personenstand im gesamten Gebiet der Union beachtet werde. Dieses Argument verwarf der EuGH jedoch mit der Begründung, dass das zuständige Organ keine Entscheidung getroffen hätte, die sich auf seine personenstandsrechtliche Lage ausgewirkt habe. ${ }^{65}$ Anders ausgedrückt: Die eingetragene Partnerschaft nach schwedischem Recht werde nicht dadurch infrage gestellt, dass für Ehepaare günstige Regelungen nicht auf sie angewandt würden; denn der Beamte $D$. bleibe auch ohne Haushaltszulage eingetragener Partner. Mit dieser Rechtsprechung wurde die persönliche Rechtsstellung auf einen rein formalen Status ohne jede weitergehende Bedeutung reduziert.

\section{b) Maruko (2008)}

Im Fall Maruko ${ }^{66}$ musste der EuGH entscheiden, ob ein eingetragener Lebenspartner nach dem Versterben seines Partners Anspruch auf Hinterbliebenenversorgung im Rahmen eines berufsständischen Versorgungssystems hat. Die betreffende „Versorgungsanstalt der deutschen Bühnen“ hatte die begehrten Leistungen versagt, weil Herr Maruko nicht überlebender Ehegatte, sondern ,nur“ eingetragener Partner des verstorbenen Arbeitnehmers war.

64 EuGH, verb. Rs. C-122/99 P und C-125/99 P, D. und Schweden gegen Dänemark und Niederlande, Slg. 2001, I-4319.

65 Ibid., Rdnr. 43.

66 EuGH, Rs. C-267/06, Maruko, Slg. 2008, I-1757. 
Ein Verstoß gegen die Richtlinie 2000/78/EG wegen Diskriminierung kommt in solchen Fällen nur in Betracht, wenn die Zahlung aus der berufsständigen Versorgungskasse zumindest „,mit Bezug auf“ ein „Arbeitsentgelt“ im Sinne von Art. 157 AEUV geleistet wird (Art. 3 Abs. 1 lit. c) und es sich nicht um Leistungen aus Sozialversicherungs- oder Sozialschutzsystemen handelt, es sei denn, diese sind dem Arbeitsentgelt gleichgestellt (Art. 3 Abs. 3 in Verbindung mit Erwägungsgrund 13). Eine solche Gleichstellung bejahte der EuGH hier, weil die Leistung aufgrund einer vormals bestehenden Beschäftigung vom früheren Arbeitgeber sowie aus den Beiträgen des Arbeitnehmers, also nicht aus der allgemeinen Staatskasse zu zahlen war. Er präzisierte dabei die Kriterien, welche Ruhegehaltsleistungen zum ,Arbeitsentgelt“" machen ${ }^{67}$ und damit dem Diskriminierungsverbot der Richtlinie unterwerfen.

Zum Erwägungsgrund 22, wonach die Richtlinie 2000/78/EG die einzelstaatlichen Rechtsvorschriften über den Familienstand unberührt lässt, stellte der EuGH sinngemäß fest: Die Mitgliedstaaten haben zwar die Regelungszuständigkeit; sie können alle möglichen Regelungsmodelle wählen. Wenn sie aber ihre Zuständigkeit ausüben, dann sind sie bei der Ausgestaltung an die Richtlinie, insbesondere das dort verankerte Diskriminierungsverbot gebunden.

Der EuGH überraschte die Fachwelt vor allem damit, dass er eine unmittelbare Diskriminierung aufgrund der sexuellen Orientierung statt einer nur mittelbaren annahm. Der Unterschied zwischen beiden Diskriminierungsformen liegt vor allem darin, dass die bloß mittelbare Differenzierung gemäß Art. 2 Abs. 2 lit. b (i) und (ii) der Richtlinie 2000/78/EG gerechtfertigt werden kann, während die unmittelbare nahezu unvermeidlich in die verbotene Diskriminierung führt. ${ }^{68} \mathrm{Ob}$ eine unmittelbare Diskriminierung vorliegt, hängt davon ab, ob eine Person wegen eines kritischen Kriteriums wie der „sexuellen Orientierung“ (Art. 1), ,in einer vergleichbaren Situation eine weniger günstige Behandlung erfährt als eine andere Person". ${ }^{69}$ Hier setzte der EuGH neue Maßstäbe.

Einerseits erklärte er es für eine Sache des vorlegenden Gerichts, ob sich der Lebenspartner in einer ,vergleichbaren Situation“ wie der eines Ehepartners befindet. Obwohl er damit scheinbar plausibel an die Regelungskompetenz der Mitgliedstaaten anknüpft, über-

67 Siehe im Ergebnis ibid., Rdnr. 48: Bestandteil des Entgelts sind solche Lohnersatzleistungen dann, wenn sie nur für eine bestimmte Gruppe von Arbeitnehmern gezahlt werden, wenn sie unmittelbar von der abgeleisteten Dienstzeit abhängen und wenn die Höhe nach den letzten Bezügen berechnet wird. Siehe auch EuGH, verb. Rs. C-124/11, C-125/11 und C-143/11, Dittrich, Klinke und Müller, Rdnr. 38. Zu sehr an deutschen Konzepten haftend Stüber, Was folgt aus „Maruko“?, NVwZ 2008, S. 752: Die Ungleichbehandlung durch Versorgungswerke, die aus einem Kapitalstock Renten zahlten, sei europarechtswidrig, umlagefinanzierte Versorgungswerke wie die der freien Berufe seien dagegen dem System der sozialen Sicherheit gleichgestellt und vom Urteil Maruko nicht betroffen.

68 Nicht überzeugend Hatje, in: Schwarze (Hrsg.), EU-Kommentar, 3. Aufl. 2012, Art. 6 EUV, Rdnr. 24, wonach die „begründete“ Besserstellung der Ehe gegenüber gleichgeschlechtlichen Partnerschaften keinen Verstoß gegen den Gleichheitssatz darstelle. Die dafür zitierte Entscheidung des EuGH (Rs. C-117/01) ist älteren Datums (7.1.2004) und betrifft eine Diskriminierung aufgrund des Geschlechts in einem Transsexuellen-Fall, in dem trotz Geschlechtsumwandlung die registerrechtliche Änderung des Geschlechts und deshalb die Eheschließung verweigert worden war.

69 EuGH, Rs. C-267/06, Maruko, Slg. 2008, I-1757, Rdnr. 66. 
zeugt diese Position nicht, weil es sich bei der vergleichbaren Situation um einen europäischen Rechtsbegriff handelt, deren Vorliegen letztendlich das europäische System - der EuGH - ,autonom“ beurteilen muss: Überlassen werden kann den staatlichen Gerichten zwar die Ermittlung aller Fakten, Umstände und nationalen Regelungen, aus denen sich das Urteil über die Vergleichbarkeit ergibt, nicht jedoch die abschließende Bewertung über die Vergleichbarkeit selbst. Indem der EuGH insoweit nicht einmal die Plausibilität überprüfen will, entzieht er sich seiner Aufgabe zur Rechtsprechung.

Andererseits hat der EuGH den Bewertungsspielraum der staatlichen Gerichte aber doch eingeschränkt, indem diese die Vergleichbarkeit immer nur in Bezug auf den konkreten Kontext, also nicht abstrakt klären dürfen. ${ }^{70}$ Im vorliegenden Fall dürfen die deutschen Gerichte also nicht abstrakt die Lebenspartnerschaft mit der Ehe vergleichen und zu dem Schluss kommen, dass die Partnerschaft bewusst anders als die Ehe geregelt sein sollte, so dass allein schon deshalb keine Vergleichbarkeit bestehe. Sie müssen vielmehr die Situation in Bezug auf die konkrete Begünstigung, hier die Hinterbliebenenversorgung, vergleichen. Sie werden dabei feststellen, dass eingetragene Lebenspartner sich ebenso wie Ehepartner rechtsverbindlich zu gegenseitiger Fürsorge verpflichtet haben und sie dies im Bereich der Hinterbliebenenversorgung, die Unterhaltsersatzfunktion hat, außerordentlich vergleichbar macht. Auf das zunehmend fragwürdige Argument, in der Ehe würden typischerweise Kinder erzogen, in der Partnerschaft nicht, ${ }^{71}$ ließ sich der EuGH gar nicht erst ein. Vielmehr hob er mit Bezug auf die Bundesrepublik Deutschland hervor, dass der deutsche Gesetzgeber durch die Novelle des Lebenspartnerschaftsgesetz vom 15. Dezember 2004 ja selbst den Willen zur schrittweisen Annäherung der eingetragenen Partnerschaft an die Ehe bekundet habe. ${ }^{72}$ Auch eine solche Selbstbindung spricht für die Vergleichbarkeit, obwohl sie ihrerseits einen pauschalen Charakter hat.

Das Urteil stieß, gerade soweit es die Annahme einer unmittelbaren Diskriminierung betrifft, auf heftige Kritik. Im Normalfall gilt nämlich nur diejenige Diskriminierung als unmittelbar, die direkt an das kritische Kriterium, hier die sexuelle Orientierung, anknüpft. Die streitgegenständliche Regelung zur betrieblichen Hinterbliebenenrente gebrauchte aber nur den Begriff des „Ehegatten“, worunter nach deutschem Rechtsverständnis eben keine eingetragenen Partner fallen. ${ }^{73}$ Spricht das nicht eher für eine mittelbare Diskriminierung, in der die Regelung ,neutral“ daherkommt und lediglich überproportional häufig eingetragene Partner nachteilig betrifft? Dann hätte sich freilich die Frage gestellt, ob Deutschland sich auf den besonderen Schutz der Ehe als rechtmäßiges Ziel im Sinne von Art. 2 Abs. 2 lit. b (i) der Richtlinie 2000/78/EG berufen könnte, um den Ausschluss von eingetragenen Partnern von der Begünstigung Hinterbliebener zu rechtfertigen. Damit wäre Art. 6 GG in den Fokus des EuGH geraten; und am Ende hätte man womöglich erörtern

70 Siehe insbes. ibid., Rdnr. 73. Noch klarer EuGH, Rs. C-147/08, Römer, Slg. 2011, I-3591, Rdnr. 42.

71 Zum „Kindererziehungsargument“ Grünberger, Die Gleichbehandlung von Ehe und eingetragener Lebenspartnerschaft im Zusammenspiel von Unionsrecht und nationalem Verfassungsrecht - Das Urteil des BVerfG zur VBL-Hinterbliebenenrente, FPR 2010, S. 206.

72 Siehe insbes. EuGH, Rs. C-267/06, Maruko, Slg. 2008, I-1757, Rdnr. 68 f.; sowie unter D.III.

73 Eine analoge Anwendung der Regelungen für die Ehe auf eingetragene Partnerschaften scheidet aus. Siehe unter D.I. sowie Grünberger, (Fn. 71), S. 203. 
müssen, ob Homosexuelle nicht auch die Ehe mit einer Person anderen Geschlechts schließen und deshalb gar nicht diskriminiert werden könnten. Solchen Rechtfertigungsübungen ist der EuGH ausgewichen, indem er eine unmittelbare Diskriminierung angenommen hat.

Diese Annahme ist aber auch rechtlich plausibel, denn der Begriff des „Ehegatten“ ist eben nicht neutral, nicht einmal scheinbar neutral wie bei der typischen mittelbaren Diskriminierung: Er schließt nicht nur überwiegend viele verpartnerte Personen, sondern alle Angehörigen dieser Gruppe aus, für deren gleichgeschlechtliches Zusammenleben, wie es Art. 8 EMRK schützt, die Ehe in Deutschland nicht offen steht. Verpartnerung steht somit stellvertretend für gleichgeschlechtliche Bindung. ${ }^{74}$ Vergleichbares sehen wir im Falle von Begriffen wie zum Beispiel „Mütter“, die ebenfalls als unmittelbar geschlechtsbezogen gelten, obwohl sie nicht explizit an das „Geschlecht“ anknüpfen. ${ }^{75}$ In diesen Fällen begründet die Anknüpfung an ein Stellvertreterkriterium anstelle des eigentlichen Kriteriums die unmittelbare Diskriminierung.

\section{c) Dittrich, Klinke und Müller (2012)}

Im Fall Dittrich, Klinke und Müller (2012) ${ }^{76}$ hatte das Bundesverwaltungsgericht dem EuGH die Frage vorgelegt, ob die Beihilfe für Beamten im Krankheitsfalle Entgeltbestandteil im Sinne von Art. 157 AEUV ist, so dass auch in diesem Bereich die Richtlinie 2000/78/EG anwendbar wäre. In dieser verbundenen Rechtssache präzisierte der EuGH den Entgeltbegriff vor allem durch die Bildung von drei Voraussetzungen:

Erstens muss der materielle Tatbestand des „Entgelts“ Art. 157 Abs. 2 AEUV erfüllt sein. Darunter fallen alle Vergütungen, die der Arbeitgeber wenigstens mittelbar aufgrund des Dienstverhältnisses gewährt, sei es aufgrund eines Arbeitsvertrags, aufgrund von Rechtsvorschriften oder freiwillig. ${ }^{77}$

Zweitens müsse die Beihilfe,,aufgrund des Dienstverhältnisses“ geleistet werden. Dieses sogenannte „kausale Element“ ergibt sich unmittelbar aus Art. 157 Abs. 2 AEUV. Hier hing die Beihilfeberechtigung davon ab, dass der Beamte ein Entgelt oder eine entsprechende Leistung bezieht.

Drittens verlangt Art. 157 AEUV, dass die Leistung vom Arbeitgeber gezahlt wird, also nicht aus dem Haushalt der Sozialversicherung, sondern im Falle der öffentlichen Verwaltung aus deren Etat für Personalausgaben.

Damit war nicht nur klargestellt, dass die Beihilfe für Beamte im Krankheitsfall, wie in der Bundesrepublik Deutschland geregelt, der Richtlinie unterfällt. Es wurden auch die Weichen dafür gestellt, einen Großteil der Leistungen für Beamte und Angestellte des öffentlichen Dienstes unter den Begriff des „Arbeitsentgelts“ zu fassen. Auch insoweit sind Differenzierungen zwischen verheirateten und „nur“ verpartnerten Personen untersagt.

74 Siehe auch ibid., S. 205.

75 Siehe etwa BVerfGE 114, 357 (364). Näheres zu den „Geschlechtersurrogaten“ Richter, (Fn. 7), S. 958.

76 EuGH, verb. Rs. C-124/11, C-125/11 und C-143/11, Dittrich, Klinke und Müller.

77 Auch zum Folgenden ibid., Rdnr. 35 ff. 


\section{Bewertung: Fortschreitender Einfluss des Europarechts auf die Rechtsordnungen der Mitgliedstaaten}

Die hier exemplarisch ausgewählten Fälle zeigen jene Grundstrukturen auf, die das Europarecht im Bereich des Umgangs mit gleichgeschlechtlichen Paarverbindungen zum immer einflussreicheren Faktor für das innerstaatliche Recht machen: Einerseits kann ein vorteilhafter Bindungsstatus (z.B. die eingetragene Partnerschaft oder die gleichgeschlechtliche Ehe) seine vorteilhaften Wirkungen nicht automatisch auch in allen anderen Mitgliedstaaten entfalten (Fall D.); andererseits wird die Vorenthaltung begünstigender Leistungen - auch gerade im Vergleich zur klassischen Ehe - dem Verbot der Diskriminierung aufgrund der sexuellen Orientierung und damit einer dichten Kontrolle unterworfen. Wichtigstes Instrument hierfür ist die Richtlinie 2000/78/EG zur Festlegung eines allgemeinen Rahmens für die Verwirklichung der Gleichbehandlung in Beschäftigung und Beruf, die alle Leistungen erfasst, welche nach den Kriterien des EuGH (Fall Dittrich, Klinke und Müller) als „Arbeitsentgelt“ gelten können. Denn sie bringt das Arbeitseinkommen im umfassenden Sinne in den Anwendungsbereich des europarechtlichen Diskriminierungsverbots. Handelt es sich um eine Leistung mit Entgeltcharakter, kommt es auf die Vergleichbarkeit der konkreten Situation mit ihrem konkreten Leistungszweck an, ohne dass sich der Mitgliedstaat abstrakt auf eine grundsätzlich gewollte Schlechterstellung der Partnerschaft gegenüber der Ehe berufen könnte (Fall Maruko). So wird mit jeder neuen Klage bzw. Vorlage an den EuGH die Lage für immer mehr Einzelleistungen aus dem Einkommensbereich geklärt.

\section{EGMR: Schalk und Kopf (2010)}

Die Rechtsprechung des Europäischen Gerichtshofs für Menschenrechte ist für europäische Staaten wie zum Beispiel Deutschland aus zweierlei Gründen erheblich: Zum Einen müssen sie die Europäische Menschenrechtskonvention als Vertragsstaaten im Rahmen des Europaratssystems beachten; zum Anderen adoptiert das grundrechtliche System der EU vermittels Art. 6 Abs. 3 EUV die EMRK-Rechte, ${ }^{78}$ an deren Bedeutung sich im Zweifel wiederum die Interpretation der Grundrechtecharta orientiert (Art. 52 Abs. 3 GrCh). Während es eine ganze Reihe einschlägiger Fälle mit Bezug zum Privatleben bzw. zur Diskriminierung aufgrund der ,,sexuellen Orientierung“(Art. 8, 14 EMRK) gibt, ${ }^{79}$ gerät erst in neuerer Zeit auch die Eheschließungsfreiheit (Art. 12 EMRK) in den Blick:

78 Siehe unter B.II.2.

79 Auf der Basis von Art. 8 EMRK: EGMR, Nr. 7525/76, Dudgeon, Series A 45 (1981); EGMR, Nr. 10581/83, Norris, Series A 142 (1988); EGMR, Nr. 15070/89, Modinos, Series A 259 (1993); EGMR, Nr. 33985/96 et al., Smith and Grady, Rep. 1999-VI. Auf der Basis von Art. 14 i.V.m. Art. 8 EMRK: EGMR, Nr. 39392/98 et al., L. and V. v. Austria, Rep. 2003-I; EGMR, Nr. 33290/96, Salgueiro da Silva Mouta, Rep. 1999-IX; EGMR, Nr.36515/97, Fretté, Rep. 2002-I; EGMR, Nr. 40016/98, Karner, Rep. 2003-IX; EGMR, Nr. 43546/02, Urt. v. 22.1.2008, E.B. v. France; EGMR, Nr. 13102/02, Urt. v. 2.3.2010, Kozak; EGMR, Nr. 29381/09 et al., Urt. v. 7.11.2013, Vallianatos et al. Zur Vergleichbarkeit dreier verschiedener Formen der Kohabitation: EGMR (GK), Nr. 13378/05, Burden, Rdnr. 58-66. Alle Urteile des EGMR sind abrufbar unter hudoc.echr.coe.int. Siehe auch EGMR, Factsheet - Sexual Orientation Issues, Januar 2014. 
Im Fall Schalk und Kopf gegen Österreich $(2010)^{80}$ ging es um die Frage, ob aus der EMRK auch ein Recht auf Eheschließung für gleichgeschlechtliche Paare folgt. Die Herren Schalk und Kopf hatten noch vor Verabschiedung des österreichischen Partnerschaftsgesetzes beim Standesamt der Stadt Wien das Aufgebot beantragt, wurden aber abgewiesen, weil das österreichische Allgemeine Bürgerliche Gesetzbuch die Eheschließung nur zwischen Personen unterschiedlichen Geschlechts zuließ. In diesem Fall gelangte der EGMR erstmals zu dem Schluss, dass Art. 12 EMRK (Eheschließungsfreiheit!) auf gleichgeschlechtliche Paare anwendbar sei, auch wenn die Entscheidung über die Einführung der gleichgeschlechtlichen Ehe bei den Staaten verbleibe. ${ }^{81}$

Das entscheidende Argument für die Anwendung der Eheschließungsfreiheit im Fall Schalk und Kopf war der Wandel in den Anschauungen, wie er sich gerade auch in der offenen Konzeption des Art. 9 GrCh niedergeschlagen hatte, der nämlich keinerlei Bezug mehr auf die verschiedenen Geschlechter nimmt. Hier rekurriert also das dem Europarat zugehörige System der EMRK auf das Rechtssystem der EU - während es sich bislang eher umgekehrt verhielt. Im Ergebnis verneinte der EGMR im Fall Schalk und Kopf die Verletzung der Eheschließungsfreiheit jedoch, weil die Ehe tief in den sozialen und kulturellen Verhältnissen der einzelnen Gesellschaften verwurzelt sei und insoweit noch große Unterschiede vorherrschten. Man könne deshalb (noch) nicht zu dem Ergebnis kommen, dass Art. 12 EMRK zur Öffnung der Ehe verpflichte. ${ }^{82}$

Eine Verletzung von Art. 14 in Verbindung mit Art. 8 EMRK wegen der womöglich zu späten Einführung der eingetragenen Partnerschaft lehnte der EGMR ebenfalls ab. Zwar erfordern Differenzierungen anhand der sexuellen Orientierung besonders schwerwiegende Gründe, ${ }^{83}$ hinsichtlich des gesetzgeberischen Timing gestand er dem betroffenen Vertragsstaat jedoch einen großen Ermessensspielraum zu. ${ }^{84}$ Auch die Differenzierung zwischen der eingetragenen Partnerschaft und der Ehe als solche verstoße angesichts des noch fehlenden Konsenses der Konventionsstaaten nicht gegen die EMRK. ${ }^{85}$ Jedoch bekannte er sich in Schalk und Kopf erstmals zu der Auffassung, dass das Zusammenleben in einer „stabilen de facto Partnerschaft“" nicht nur als eine Form der Gestaltung des „Privatlebens“, sondern als „Familienleben“ im Sinne von Art. 8 EMRK geschützt sei.

Die vorsichtige Öffnung, welche das Urteil bewirkt, stellt sich als Fortsetzung der im Transsexuellen-Fall der Christine Goodwin (2002) begründeten Rechtsprechung dar. Schon hier hatte der EGMR klargestellt, dass der Geschlechtsbegriff in Art. 12 EMRK nicht rein biologisch verstanden werden dürfe. ${ }^{86}$ Wenn aber letztlich jede Person im heiratsfähigen Alter, sei es aus der Gruppe „Männer“ oder aus der Gruppe „Frauen“, ein Recht auf Eheschließung hat und Art. 12 EMRK zudem keine biologisch fundierte Zuordnung zu einer der beiden Gruppen erfordert, macht es keinen Sinn mehr, das Begehren nach Heirat

80 EGMR, Nr. 30141/04, Urt. v. 24.6.2010, Schalk and Kopf.

81 Ibid., Rdnr. 61.

82 Ibid., Rdnr. $63 \mathrm{f}$.

83 Ibid., Rdnr. 97: „differences based on sexual orientation require particularly serious reasons by way of justification".

84 Ibid., Rdnr. 106.

85 Ibid., Rdnr. 107-110.

86 EGMR, Nr. 28957/95, Urt. v. 11.7.2002, Christine Goodwin, Rdnr. 100-104. 
innerhalb desselben biologischen Geschlechts von vornherein für nicht geschützt zu halten. ${ }^{87}$

In der Begründung überzeugt die Entscheidung nicht, da Art. 12 EMRK keinen absoluten Regelungsvorbehalt zugunsten der Vertragsstaaten enthält: Er gesteht ihnen die Regelungskompetenz hinsichtlich der „Ausübung dieses Rechts“ (,according to the national laws governing the exercise of this right"), aber nicht zur Vorenthaltung desselben zu. Eine staatliche Regelung, welche die Ehe auf Personen gleicher Hautfarbe beschränken würde, würde der EGMR selbstverständlich nicht akzeptieren und keineswegs der Regelungsmacht der Staaten anheimstellen. Da die jeweiligen Verbote der Diskriminierung anhand solcher Kriterien aber grundsätzlich gleichwertig sind, begründet der Hinweis auf die Lage der Dinge (,as matters stand“) ${ }^{88}$ nicht, warum die Ungleichbehandlung im Kontext sexueller Orientierung so viel weiter gehen darf als im Falle von Hautfarbe oder sogenannter „Rasse“. 89

Ungeachtet dessen ist in der Anerkennung homosexueller Paare mit Kindern als „Familie" eine enorme Aufwertung der homosexuellen Lebensgemeinschaft zu sehen. Sie wird sich vor allem auf die Prüfung der Verhältnismäßigkeit von Beschränkungen und Zurücksetzungen auswirken, d.h. dort den Kontrollmaßstab verschärfen.

\section{Umsetzungsprobleme - am Beispiel des deutschen Rechts}

\section{Aufgabe des Abstandsgebots und Konservierung eines normativen Ehebegriffs}

Im deutschen Recht ist die Diskussion um das Verhältnis zwischen Lebenspartnerschaft und Ehe noch immer von der Figur des Abstandsgebots und ihren Nachwirkungen geprägt. ${ }^{90}$ Die betreffende Doktrin leitet aus dem besonderen Schutzauftrag des Staates aus Art. 6 GG zugunsten der Ehe ab, dass Lebensverbindungen anderer Art als die Ehe auf Abstand zu dieser zu halten seien, d.h. nicht dieselben Vorzüge wie diese genießen, in jedem Falle aber schlechter behandelt werden dürften. ${ }^{91}$ Prämisse hierfür ist wiederum, dass sich der Ehebegriff des Art. 6 GG nur auf die Ehe zwischen einem Mann und einer Frau bezieht. Obwohl das Bundesverfassungsgericht das Abstandsgebot inzwischen klar

87 Wiemann, (Fn. 34), S. 410, erkennt in Schalk und Kopf ebenso wie in Goodwin eine Verletzung des Kernbereichs von Art. 12 EMRK, da die betroffenen Personen in beiden Fällen entweder tatsächlich oder rechtlich und damit an jeglicher Eheschließung gehindert seien.

88 EGMR, Nr. 30141/04, Urt. v. 24.6.2010, Schalk and Kopf, Rdnr. 61.

89 Wiemann, (Fn. 34), S. 411 ff., spricht von einer „Flucht in den Beurteilungsspielraum“, der losgelöst von der gebotenen Rechtfertigungsprüfung und unzureichend begründet sei. Siehe auch das Sondervotum der Richter Rozakis, Spielmann und Jebens zu EGMR, Nr. 30141/04, Urt. v. 24.6.2010, Schalk and Kopf.

90 Siehe etwa Hofmann, in: Schmidt-Bleibtreu/Hofmann/Hopfauf, GG, 11. Aufl. 2008, Art. 6, Rdnr. 18 ff., wonach die eingetragene Lebenspartnerschaft als Ehe unter lediglich anderem Namen mit dem verfassungsrechtlichen Leitbild der Ehe unvereinbar sei. Siehe auch Frenz, Eheschutz ade?, BVerfG stärkt gleichgeschlechtliche Paare, NVwZ 2013, S. 1202, wonach das Abstandsgebot eine Möglichkeit von mehreren bleibe, dem Willen der Schöpfer des Grundgesetzes in Bezug auf Art. 6 Abs. 1 GG Rechnung zu tragen.

91 In diese Richtung noch BVerfG (Kammer), NJW 2008, 209: Art. 6 Abs. 1 GG enthalte ein ,Differenzierungsgebot". 
verabschiedet hat ${ }^{92}$ und die Ausgestaltung des Instituts „Ehe“ weitgehend dem einfachen Gesetzgeber überlässt, betrachtet es die heterosexuelle Natur der Verbindung doch nach wie vor als begriffsprägenden, festen Kernbestand der Ehe, der sogar dem gesellschaftlichen Wandel entzogen sein soll. ${ }^{93}$ Dem entsprechend lehnt auch die Fachgerichtsbarkeit eine analoge Anwendung des Begriffs „Ehegatte“ oder „Ehepartner“ auf Lebenspartner ab. ${ }^{94}$

Kennzeichnend für die deutsche Rechtslage ist somit eine Entkoppelung von Ehe und Familie, die zu einer relativ offenen Konzeption des Familienbegriffs geführt hat, während der Ehebegriff für gleichgeschlechtliche Paare verschlossen bleibt. Soweit dies nach Auffassung des Bundesverfassungsgerichts sogar auf Dauer oder gar Ewigkeit Bestand haben soll, beschwört es Bewahrung, Zuordnung und Zugehörigkeit: Zum Gehalt der Ehe „gehört" danach etwas, das sich ungeachtet des gesellschaftlichen Wandels und folgender Änderungen in der rechtlichen Gestaltung „,bewahrt“ und durch das Grundgesetz seine „Prägung bekommen“" habe, nämlich dass die Ehe einen Mann und eine Frau (- also nicht zwei Frauen oder zwei Männer) zur dauerhaften Lebensgemeinschaft vereinige. ${ }^{95}$

Kann damit die Erosion (negativ) oder Öffnung (positiv) des Ehebegriffs, wie er sich in etlichen Staaten der Welt schon abzeichnet, ${ }^{96}$ aufgehalten werden? In der Tat scheint das Bundesverfassungsgericht den Versuch zu unternehmen, den Rechtsbegriff „Ehe“ vom sprachlich-gesellschaftlichen Ehebegriff ${ }^{97}$ abzukoppeln und dadurch in seinem ursprünglichen Gehalt zu konservieren. Der mit dem Willen der Verfassungsschöpfer verknüpfte juristische Ehebegriff soll so gegen künftige Veränderungen der Rechtskultur - insbesondere auch unter dem Einfluss internationaler Entwicklungen - immunisiert werden. Dabei spielt das Bundesverfassungsgericht auch mit einer syntaxbedingten Zweideutigkeit: „Zum Gehalt der Ehe, wie er sich ungeachtet des gesellschaftlichen Wandels [...] bewahrt und durch das Grundgesetz seine Prägung bekommen hat" ${ }^{\prime 9}{ }^{9}$ kann bedeuten, dass der besagte

92 BVerfGE 105, 313 (348) - LebenspartnerschaftsG: „Aus der Zulässigkeit, in Erfüllung und Ausgestaltung des Förderauftrags die Ehe gegenüber anderen Lebensformen zu privilegieren, lässt sich jedoch kein in Art. 6 Abs. 1 GG enthaltenes Gebot herleiten, andere Lebensformen gegenüber der Ehe zu benachteiligen." Es gelte zwar ein Verbot, mit der Ehe austauschbare Institute mit derselben Funktion zu schaffen, Lebenspartnerschaft und Ehe seien aber schon wegen ihres unterschiedlichen Adressatenkreises nicht austauschbar.

93 Siehe BVerfGE 105, 313 (345) - LebenspartnerschaftsG: „Zum Gehalt der Ehe, wie er sich ungeachtet des gesellschaftlichen Wandels und der damit einhergehenden Änderungen ihrer rechtlichen Gestaltung bewahrt und durch das Grundgesetz seine Prägung bekommen hat, gehört, dass sie die Vereinigung eines Mannes mit einer Frau zu einer auf Dauer angelegten Lebensgemeinschaft ist, [...].“ Noch schärfer BVerfGE 121, 175 (195) - Transsexuellengesetz: „Das gesetzgeberische Anliegen, das Rechtsinstitut der Ehe, die unter dem besonderen Schutz von Art. 6 Abs. 1 GG steht, als Form des rechtlich abgesicherten Zusammenlebens ausschließlich Mann und Frau, also Partnern verschiedenen Geschlechts, vorzubehalten, ist von hohem Gewicht." (Hervorhebung durch Verf.).

94 Siehe BGH, NJW-RR 2007, 1441; Grünberger, (Fn. 71), S. 203.

95 Siehe Fn. 93.

96 Siehe unter A.III.

97 Beck, Gleichstellung durch Öffnung der Ehe, FPR 2010, S. 225, weist darauf hin, dass der Duden unter dem Begriff der „Ehe“ die gleichgeschlechtliche Ehe bereits als neue Bedeutungsebene thematisiert (m.N.).

98 Siehe Fn. 93. 
Gehalt der Ehe sich jedenfalls bislang bewahrt hat - oder aber, dass die Bewahrung fortdauert, je nach dem, ob man das abschließende Hilfsverb „hat“ auch auf „,bewahrt“ oder nur auf die Formulierung „Prägung bekommen“ bezieht, es also in der Perfekt- oder Präsensform gebraucht ist. Letztlich erscheint die Position des Bundesverfassungsgerichts aber unabhängig davon nur solange haltbar wie ihre Kernaussage, wonach sich ein bestimmter Inhaltskern der Ehe bewahrt (habe), noch zutrifft, also von der Rechtsgemeinschaft ganz überwiegend geteilt wird. Sollte das eines Tages nicht mehr der Fall sein, bleibt nur noch das Festhalten am Willen der Verfassungsschöpfer, das aber nach den allgemeinen Grundsätzen der Verfassungsinterpretation niemals genügt, um einen Bedeutungswandel dauerhaft auszuschließen. ${ }^{99}$

\section{Begrenzung des Erfordernisses der Verschiedengeschlechtlichkeit auf den Zeitpunkt des Eheschlusses}

2008 musste das Bundesverfassungsgericht erstmals eine gleichgeschlechtliche Ehe anerkennen. ${ }^{100}$ Es handelte sich allerdings um einen außergewöhnlichen Fall, in dem der Ehemann eine Geschlechtsumwandlung zur Frau durchführen lassen und seine bisherige Ehefrau mit ihm auch weiterhin verheiratet bleiben wollte. Die damalige Regelung, welche die Geschlechtsumwandlung von der vorherigen Ehescheidung abhängig machte, bewertete das Bundesverfassungsgericht als unverhältnismäßig. Die rechtliche Basis hierfür war, dass das Paar sich auch weiterhin auf Art. 6 Abs. 1 GG berufen konnte, da für dessen Schutzwirkung allein entscheidend sei, dass die Ehe jedenfalls ursprünglich zwischen verschiedengeschlechtlichen Partnern geschlossen worden war. ${ }^{101}$ Die Folge dieser Entscheidung ist, dass es die gleichgeschlechtliche Ehe - in verschwindend geringem Ausmaß - nun auch innerhalb der deutschen Rechtsordnung gibt.

99 Siehe insbes. BVerfGE 62, 1 (45) - Bundestagsauflösung I: „Das Bundesverfassungsgericht hat wiederholt ausgesprochen, daß die Gesetzesmaterialien mit Vorsicht, nur unterstützend und insgesamt nur insofern herangezogen werden sollen, als sie auf einen, objektiven Gesetzesinhalt schließen lassen' [...]. [...] Ob diese Grundsätze auch uneingeschränkt für die Auslegung von Verfassungsnormen gelten können, mag dahinstehen. Denn mehr als die Interpretation der Gesetze hat die der Verfassung mit dem Problem der Offenheit des Normtextes zu tun, weil die Verfassung der aufgegebenen politischen Einheit des Staates zu dienen bestimmt ist [...]. [...] Insbesondere hinsichtlich des organisatorischen Teils der Verfassung, [...], wird die Aufgabe der Verfassungsinterpretation dahin verstanden, wechselnden Gestaltungsmöglichkeiten Raum zu lassen [...]. [...] Dennoch kann die Entstehungsgeschichte einer solchen Norm nicht völlig unberücksichtigt bleiben, insbesondere dann nicht, wenn sich für ihre Auslegung feste Grundsätze noch nicht haben bilden können [...]. Ausschlaggebende Bedeutung kommt den Verfassungsmaterialien allerdings in der Regel nicht zu [...].“

100 BVerfGE 121, 175 (175 ff.) - Transsexuellengesetz.

101 BVerfGE 121, 175 (198) - Transsexuellengesetz: „Auch die Ehe von Ehegatten, in denen einer von ihnen während der Ehezeit seine Transsexualität entdeckt oder offenbart und entsprechend seinem empfundenen oder während der Ehe durch operative Eingriffe gewandelten Geschlecht lebt, fällt unter den Schutz des Art. 6 Abs. 1 GG. Sie ist als Ehe zwischen einem Mann und einer Frau rechtmäßig zustandegekommen und hat bei ihrem Eingehen allen Merkmalen entsprochen, die den Gehalt der Ehe ausmachen." 


\section{Fehlen eines gesetzgeberischen Konzepts und „scheibchenweiser“ Nachvollzug des Europarechts}

Obwohl das 2001 in Kraft getretene Lebenspartnerschaftsgesetz ${ }^{102}$ den Lebenspartnern die volle Fürsorgelast füreinander auferlegte, ohne ihnen zugleich die vollen Ehegattenrechte zu übertragen, wurde es von den Bundesländern Bayern, Sachsen und Thüringen mittels Normenkontrollklage angegriffen. Die für die Kläger unerwünschte Folge war allerdings, dass das Bundesverfassungsgericht mit Grundsatzentscheid von 2002 die VerfassungsmäBigkeit des konkreten Gesetzes wie auch die Verfassungsmäßigkeit einer Gleichstellung von Lebenspartnerschaft und Ehe bestätigte und sich dabei auch deutlich vom Abstandsgebot distanzierte. ${ }^{103}$ Dies ermunterte wiederum den Gesetzgeber zu einer Weiterentwicklung der eingetragenen Partnerschaft, ${ }^{104}$ wobei die Gesetzesnovelle explizit bezweckte, „künstliche Unterscheidungen zwischen Ehe und Lebenspartnerschaft“ zu beseitigen und „weitgehende Angleichungen des Rechts der Lebenspartnerschaft an das Recht der Ehe“ vorzusehen. ${ }^{105}$ Dies wurde vom vorlegenden Gericht in der Rechtssache Maruko dann als Wille zur bzw. Konzept der ,schrittweisen Annäherung“ interpretiert, worin der EuGH wiederum ein Indiz für die Vergleichbarkeit der Situation von eingetragenen Partnern im Verhältnis zu Ehepartnern sah. ${ }^{106}$

Seit dem Urteil Maruko war klar, dass immer neue Fallkonstellationen der Schlechterstellung von eingetragenen Lebenspartnern gegenüber Ehepartnern auf den Rechtsweg gelangen und mit hoher Wahrscheinlichkeit zugunsten der betroffenen Lebenspartner ausgehen würden. ${ }^{107} \mathrm{Zu}$ einer grundlegenden Lösung zugunsten homosexueller Paare konnten sich aber weder die Große Koalition (CDU/CSU und SPD) in der 16. Legislaturperiode (2005-09) noch die CDU/CSU-FDP-Koalition in der 17. Legislaturperiode (2009-13) entschließen. ${ }^{108}$ Dies führt dazu, dass der deutsche Gesetzgeber seither zu immer neuen Nachbesserungen an immer weiteren Einzelstellen des Rechtssystems getrieben wird, je nachdem, wo es gerade zum Streit mit Betroffenen kommt.

Auch dem Bundesverfassungsgericht war das Heft des Handelns aus der Hand genommen, - solange es jedenfalls der These folgte, dass Voraussetzung für das Eingehen einer Ehe oder Lebenspartnerschaft allein ,die Geschlechtskombination der Partner, nicht eine bestimmte sexuelle Orientierung" sei. ${ }^{109}$ Ein durchgreifender Wandel der Rechtsprechung setzte erst mit dem Beschluss vom 7. Juli 2009 zur betrieblichen Hinterbliebenenversorgung im öffentlichen Dienst ein, mit dem sich der 1. Senat des Bundesverfassungsgerichts der Auffassung anschloss, dass es in den fraglichen Fällen eben doch um nichts Anderes

102 BGB1. 2001 I, 266; RegE BT-Drs. 14/4545; Rechtsausschuss BT-Drs. 14/3751 und 14/4550.

103 BVerfGE 105, 313 (345) - LebenspartnerschaftsG. Zum Abstandsgebot oben D.I.

104 BGBl. 2004 I, 3396; RegE BT-Drs. 15/3445; Rechtsausschuss BT-Drs. 15/4052.

105 Siehe Gesetzesbegründung, RegE BT-Drs. 15/3445, S. 1.

106 EuGH, Rs. C-267/06, Maruko, Slg. 2008, I-1757, Rdnr. 68 f. Siehe im Ergebnis bereits unter C.I.1.b).

107 Siehe z.B. EuGH, Rs. C-147/08, Römer, Slg. 2011, I-3591.

108 Vgl. zur gesetzgeberischen Entwicklung von der 14. bis zur 16. Legislaturperiode Beck, (Fn. 97), S. $220 \mathrm{ff}$.

109 So noch BVerfG (1. Kammer des 2. Senats), NJW 2008, 209 (211), unter Bezugnahme auf BVerfGE 115, 1 (16); BGH NJW-RR 2007, 1441. 
als eine Anknüpfung an die sexuelle Orientierung geht, ${ }^{110}$ und sich damit von entgegenstehenden Kammerbeschlüssen des 2. Senats absetzte. ${ }^{111}$

Seither gilt auch in Bezug auf die Schlechterstellung von eingetragenen Partnern die Regel, dass die Anforderungen an die Rechtfertigung einer Ungleichbehandlung von Personengruppen im Sinne von Art. 3 Abs. 1 GG umso strenger sind, je mehr dabei an Persönlichkeitsmerkmale angeknüpft wird, die - wie zum Beispiel die sexuelle Orientierung den Merkmalen des Art. 3 Abs. 3 GG ähneln und zur Diskriminierung einer Minderheit führen können; ${ }^{112}$ es gilt also ein strenger Kontrollmaßstab. ${ }^{113}$ Dem zufolge reicht auch im deutschen Recht der Hinweis auf eine generelle Absicht des Gesetzgebers, die Ehe fördern zu wollen, bzw. die Existenz einer Verfassungsnorm mit privilegierender Tendenz (Art. 6 Abs. 1 GG) nicht mehr aus, um eine konkrete Schlechterstellung von eingetragenen Partnern gegenüber Ehepartnern zu rechtfertigen. Ebenso wenig taugt das Argument einer Koppelung von Ehe und Kindern nicht mehr als genereller Differenzierungsgrund. Denn angesichts vieler Ehen ohne Kinder und von zunehmend mehr Lebensgemeinschaften mit Kindern geht die neue Rechtsprechung des Bundesverfassungsgerichts von einem insoweit gleichartigen Schutz der Familie aus, ${ }^{114}$ während für die Ehe an und für sich nur noch die Idee des Solidarsystems als prägendes Element verbleibt. Hierin allein liegt fortan das öffentliche Interesse an der Ehe. ${ }^{115}$ Entscheidend ist demnach, ob die eingetragene Lebenspartnerschaft in puncto Entgeltcharakter, Honorierungscharakter oder Versorgungscharakter gerade im betroffenen Regelungskontext der Ehe vergleichbar ist. ${ }^{116}$ So wird im Ergebnis ein Gleichklang mit der Rechtsprechung des EuGH hergestellt, wobei das Bundesverfassungsgericht sogar in seltener Ausdrücklichkeit auf den entsprechend strengen Prüfungsmaßstab im Europarecht (EU-Grundrechte und EMRK) verweist, den es nun mehr verspätet anhand des Grundgesetzes nachvollzieht. ${ }^{117}$

Das Einlenken des Bundesverfassungsgerichts stieß teilweise auf heftige Kritik. Dem Bundesverfassungsgericht wurde nicht nur vorgeworfen, die letzten Statusunterschiede zwischen Lebenspartnern und Ehepartnern eingeebnet und damit das Verfassungsgebot aus Art. 6 Abs. 1 Alternative 1 GG „,durch Richterspruch endgültig aufgehoben“ zu haben; das Verlangen nach einem sachgerechten Grund für die Differenzierung schaffe eine bloße „Scheinrationalität“, während die Wertentscheidung des Art. 6 Abs. 1 GG gar nicht auf

110 BVerfGE 124, 199 (199 ff.) - VBL-Hinterbliebenenrente.

111 Siehe im Ergebnis von Roetteken, Anmerk. zu BVerfG 1. Senat, Beschl. v. 7.7.2009, juris PraxisReport - ArbR 48/2009, Anm. 2. Bruns, (Fn. 32), S. 1931, spricht vom ,Streit unter den Senaten“.

112 St.Rspr. Siehe statt Vieler BVerfG, Beschl. v. 6.6.2013, 2 BvR 909/06 et al., Rdnr. 77 - Ehegattensplitting (m.w.N.).

113 BVerfGE 124, 199 (220) - VBL-Hinterbliebenenrente.

114 Grünberger, (Fn. 71), S. 206, spricht von einer „Entkoppelung“ von Ehe und Familie.

115 Siehe BVerfGE 124, 199 (225) - VBL-Hinterbliebenenrente: „,Die Rechtfertigung der Privilegierung der Ehe, und zwar auch der kinderlosen Ehe, liegt, insbesondere wenn man sie getrennt vom Schutz der Familie betrachtet, in der auf Dauer übernommenen, auch rechtlich verbindlichen Verantwortung für den Partner."

116 Vgl. Wiemann, Rosige Aussichten für die Gleichstellung gleichgeschlechtlicher Lebenspartner mit Ehegatten?, NJW 2010, S. 1429.

117 BVerfGE 124, 199 (220) - VBL-Hinterbliebenenrente. 
Sachlichkeit überprüfbar sei. ${ }^{118}$ Entscheidend ist allerdings, welche Wertentscheidung dem Grundgesetz heute zu entnehmen ist. Nach hier vertretener Auffassung kann der Ehebegriff nicht nur zeitgerecht und europarechtskonform interpretiert und fortentwickelt werden, sondern hält das Grundgesetz auch jede Überprüfung auf Rationalität aus. Das Eingeständnis, eine sachliche Prüfung der Notwendigkeit von Schlechterstellungen am konkreten Sachverhalt nicht vornehmen zu können, ist geradezu Indiz dafür, dass eine aus Art. 6 Abs. 1 GG vermeintlich folgende Wertentscheidung sich nicht in die rechtsstaatliche, am Verhältnismäßigkeitsprinzip orientierte Verfassungsordnung fügt.

Auch von einem verfassungsrechtlichen Systemwandel kann keine Rede sein, weil das Bundesverfassungsgericht nicht etwa jetzt „Art. 6 I GG im Lichte von Art. 3 I GG versteht", ${ }^{119}$ während es zuvor Art. 3 GG im Lichte von Art. 6 GG verstanden hätte. Vielmehr hat das Bundesverfassungsgericht - durch Bezugnahme auf die konkrete Streitfrage lediglich seinen Kontrollmaßstab verfeinert, wie man dies von einem elaborierten Rechtsstaat erwartet. Dass gerade keiner Verfassungsvorschrift von vornherein der Vorrang über die andere eingeräumt werden sollte, bezeugt das Bundesverfassungsgericht selbst, indem es ausführt, dass sich Art. 3 und Art. 6 GG an jeweils unterschiedliche Adressatenkreise richteten und daher nicht ,austauschbar“ seien: ${ }^{120}$ Können beide Normen nicht auf denselben Sachverhalt angewandt werden, stellt sich auch die Frage einer vorrangigen Anwendbarkeit der einen Norm über die andere nicht.

Dass die Rechtsprechung auch weiterhin reparierend tätig werden muss, ist vorprogrammiert, weil das Lebenspartnerschaftsgesetz selektiv nur auf bestimmte BGB-Normen zur Ehe verweist, auf andere dagegen nicht. So fehlt zum Beispiel in $\S 15$ Abs. 2 Satz 2 LPartG eine Verweisung auf die Regelung des $§ 1318$ BGB mit der Folge, dass der arglistig täuschende Partner bei einer späteren Aufhebung der Partnerschaft Unterhalt verlangen kann. ${ }^{121}$ Ferner wird der arglistig getäuschte Lebenspartner vom überlebenden Partner beerbt, wenn das Verfahren auf Aufhebung der Partnerschaft nur anhängig, aber noch nicht rechtskräftig beendet war, ${ }^{122}$ und so weiter und so fort. Während in diesen Fällen oft nicht klar ist, ob es sich um gesetzgeberische Absicht oder handwerkliche Fehler handelt, finden sich auch Beispiele für die gewollte Differenzierung. So musste erst das Bundesverfassungsgericht feststellen, dass es gleichheitswidrig ist, die gemeinsame Adoption eines fremden Kindes durch Lebenspartner aus Gründen des Kindeswohls zu versagen, wenn eine Stiefkindadoption ${ }^{123}$ ohne dieselben Bedenken zulässig ist. ${ }^{124}$ Auf etlichen weiteren

118 Hillgruber, Anmerkung zu BVerfG, Beschl. v. 7.7.2009, JZ 2010, S. 41 ff.

119 Grünberger, (Fn. 71), S. 205.

120 Siehe BVerfGE 105, 313 (350 f.). Kritisch zur „Aliud-Argumentation“ des BVerfG Zuck, Die verfassungsrechtliche Gewährleistung der Ehe im Wandel des Zeitgeistes, NJW 2009, S. 1452, der stattdessen quantitativ argumentiert: Vergleichbare Einrichtungen für „Randgruppen“ könnten die Ehe nicht gefährden.

121 Siehe Muscheler, Die Reform des Lebenspartnerschaftsrechts, FPR 2010, S. 230.

122 Siehe ibid., S. 231.

123 Seit 2005 darf der gleichgeschlechtliche Partner ein in die Partnerschaft mitgebrachtes Kind des anderen Partners adoptieren ( 99 Abs. 7 LPartG).

124 BVerfG, Urt. v. 19.2.2013, 1 BvL 1/11 und 1 BvR 3247/09, NJW 2013, 847 ff. A.A. Schweizerisches Bundesgericht, BGE 137 III 241. In diesem Falle wurde allerdings die Frage der Vereinbarkeit mit Völkerrecht offen gelassen. 
Feldern werden eingetragene Partnerschaften immer noch auf Abstand zu den Ehegatten gehalten, zum Beispiel im Steuerrecht, ${ }^{125}$ wo sich nach dem Beschluss des Bundesverfassungsgerichts vom 7. Mai 2013 zum Ehegattensplitting allerdings ein grundlegender Wandel abzeichnet, ${ }^{126}$ oder im Beamtenrecht. ${ }^{127}$ Teile der Literatur sehen hier das „Prinzip der Willkür"128 walten.

In den meisten verbleibenden Problemfällen wird das Bundesverfassungsgericht auf der Grundlage des verschärften Prüfungsmaßstabes zu Art. 3 Abs. 1 GG in der Lage sein, Schlechterstellungen zu eliminieren, soweit diese angesichts des Fürsorge- und Versorgungscharakters der eingetragenen Partnerschaft sachlich nicht zu rechtfertigen sind. ${ }^{129}$ Deshalb wird vertreten, dass das Bundesverfassungsgericht schon jetzt die eingetragene Lebenspartnerschaft mit der Ehe gleichgesetzt habe. ${ }^{130}$ Doch trägt die Rechtsprechung dem Europarecht immer nur sukzessiv, d.h. von Problem zu Problem anhand des Einzelfalls Rechnung, während es an einer grundsätzlichen Lösung durch den Gesetzgeber fehlt. Ein Versuch der Fraktionen von SPD und Grünen vom März 2013, die Ehe durch Ergänzung des $\S 1353$ BGB auch für gleichgeschlechtliche Personen zu öffnen, ${ }^{131}$ ist im Bundestag an der damaligen Mehrheit von CDU/CSU und FDP gescheitert.

\section{E. Analyse und Prognose: Ist die Öffnung der Ehe unausweichlich?}

\section{Problem- und Entwicklungsdimensionen}

\section{Gleichgeschlechtliche Ehe und Zukunft der Gesellschaft}

Zur Debatte steht die grundlegende Veränderung einer tradierten Institution, was auf entsprechend heftige Abwehrreaktionen trifft: Bedient die gleichgeschlechtliche Ehe einen

125 Siehe zur (gescheiterten) Gesetzesinitiative einiger Länder im Bundesrat, die „Gleichstellung der Lebenspartnerschaft mit der Ehe im Einkommensteuerrecht" zu erreichen: BR-Drs. 137/13 v. 21.2.2013. Zur Versteuerung erbrechtlicher Ansprüche BVerfGE 105, 313 (355).

126 Siehe Entwurf (der Bundesregierung) eines Gesetzes zur Anpassung steuerlicher Regelungen an die Rechtsprechung des Bundesverfassungsgerichts vom 5.5.2014, BT-Drs. 18/1306, mit Bezug auf BVerfG, Beschl. v. 7.5.2013, 2 BvR 909/06 et al. - Ehegattensplitting.

127 Siehe Muscheler, (Fn. 121), S. 232.

128 Siehe ibid., S. 232. Siehe auch Classen, Die Lebenspartnerschaft im Beamtenrecht, FPR 2010, S. 200 ff., der mit Blick auf das Beamtenrecht die Gleichstellung von Ehe und Lebenspartnerschaft fordert.

129 Siehe etwa BVerfG, Beschl. v. 7.7.2009, 1 BvR 1164/07 - VBL-Hinterbliebenenrente; BVerfG, Beschl. v. 17.8.2010, 1 BvR 611/07 et al. - Erbschaftsteuer- und Schenkungsteuer; BVerfG, Beschl. v. 19.6.2012, 2 BvR 1397/09 - beamtenrechtlicher Familienzuschlag (anders noch BVerfG (1. Kammer des 2. Senats)), NJW 2008, 209 ff.; BVerfG, Beschl. v. 18.7.2012, 1 BvL 16/11 - Grunderwerbsteuer; BVerfG, Beschl. v. 7.5.2013, 2 BvR 909/06 et al. - Ehegattensplitting.

130 Siehe von der Tann, Entwicklungen in der Rechtsstellung eingetragener Lebenspartnerschaften, FamFR 2012, S. 198.

131 Entwurf eines Gesetzes zur Einführung des Rechts auf Eheschließung für Personen gleichen Geschlechts, BT-Drs. 17/12677 v. 12.3.2013. Vorgeschlagene Neufassung des $§ 1353$ Abs. 1 Satz 1 BGB: „Die Ehe wird von zwei Personen verschiedenen oder gleichen Geschlechts auf Lebenszeit geschlossen.“ 
individualistischen „Zeitgeist“, der Ehe und Familie und so „die Zukunftsfähigkeit unserer europäischen Gesellschaft schwächt"? ${ }^{132}$ Diese und ähnliche Stellungnahmen präzisieren allerdings nicht, welche Art von Einflussauf die Zukunftsaussichten der Gesellschaft zu befürchten wäre - und welche Zukunft eigentlich gewünscht ist: Wird die Gesellschaft weniger Kinder haben, wenn der Gesetzgeber neben der eingetragenen Partnerschaft auch die Ehe für gleichgeschlechtliche Paare öffnet? Oder ist gleich Beides abzulehnen? Wer Individualismus in Verbindung mit Anti-Diskriminierungspolitiken zum Vorwurf macht oder „Selbstverwirklichung“ als Egoismus-Formel gebraucht, zeigt Unbehagen gegenüber einer Minderheitenkultur, welche die Traditionen und Homogenitätserwartungen einer Mehrheit infrage stellt. ${ }^{133}$ Der proklamierte Kampf gegen den Individualismus unterschätzt jedoch das Potential des freiheitlichen Staates, der sich gerade in der Zulassung maximal möglicher Individualität und Gruppenvielfalt bewährt. Demgegenüber führt die Betonung der Interessen der Gemeinschaft gegenüber den Individualinteressen nicht zu mehr, sondern weniger Freiheit für Einzelne, wie der Streit um die kulturelle Relativität der Menschenrechte eindrücklich zeigt. Entscheidend ist letztlich jedoch die Frage nach dem Bestehen einer Wahlmöglichkeit, ob das Eingehen einer gleichgeschlechtlichen Paarverbindung also Freiheitsbetätigung im Sinne des Bestehens einer verschiedengeschlechtlichen Alternative ist oder die Anerkennung des Andersseins durch Gleichstellung zur Debatte steht. Während die Rede vom individualistischen Zeitgeist persönliche Beliebigkeit und bloßes „Lifestyle“-Interesse im Fluss einer wandelbaren Mode suggeriert, ist die außergewöhnliche sexuelle Orientierung doch - vergleichbar dem Geschlecht - ein veranlagtes Merkmal der Person, das man allenfalls unterdrücken und verbergen, aber nicht verändern oder auf Andere übertragen kann. ${ }^{134}$

\section{Die grundrechtliche Dimension}

Ist im europäischen Recht, etwa in Art. 21 Abs. 1 GrCh, der Weg zur „HomosexuellenEhe“" vorgezeichnet? ${ }^{135}$ Obwohl es heute noch keine eindeutige Antwort auf die Frage gibt, lassen sich zwei große Problemdimensionen mit ihren jeweils eigenen Tendenzen unterscheiden. Von zentraler Bedeutung ist zunächst die grundrechtliche Dimension, die Fragen des Diskriminierungsverbots und der Eheschließungsfreiheit umschließt. Das europarechtliche Diskriminierungsverbot reicht so weit, wie die Vergleichbarkeit der konkreten Situation, in Bezug auf die differenziert wird. Zwar soll die Beurteilung der Vergleichbarkeit nach Auffassung des EuGH mitgliedsstaatlichen Instanzen obliegen; diese dürfen die Lebenspartnerschaft aber nicht mehr pauschal für unvergleichbar mit der Ehe erklären, etwa

132 Tettinger/Geerlings, Ehe und Familie in der europäischen Grundrechtsordnung, EuR 2005, S. 420, unter Bezug auf das insoweit missverstandene Werk von Lewis, The Ende of Marriage? - Individualism and Intimate Relations, 2001.

133 Extrem zugespitzt Hillgruber, Wo bleibt die Freiheit der anderen?, FAZ Nr. 44 v. 21.2.2014, S. 7, der die Freiheit propagiert, ,die Homosexualität für moralisch fragwürdig und homosexuelle Praxis für anstößig“" zu halten, und dafür Minderheitenschutz reklamiert. Das verkennt die Gefährdungslage und die Verteilung des Schutzbedarfs.

134 Siehe unter A.II.

135 Tettinger/Geerlings, (Fn. 132), S. 426. 
weil die Ehe verfassungsrechtlich privilegiert ist. Indem der EuGH auf die konkrete Vergleichssituation abstellt, verengt er den Kontrollmaßstab. Grundlegend hierfür ist eine „Entkoppelung“ von Ehe und Familie, die nunmehr streng zwischen dem Schutz der Familie und dem Schutz der Ehe unterscheidet und zugleich die Kindererziehungsfunktion der Ehe als pauschales Privilegierungsargument entkräftet. Eine solche Entkoppelung lässt sich, wie gezeigt, sowohl für den international- und europarechtlichen Bereich als auch für das deutsche Verfassungsrecht beobachten. Soweit es die Eheschließungsfreiheit betrifft, spricht sowohl aus Art. 9 GrCh als auch aus der jüngsten Rechtsprechung des EGMR zu Art. 12 EMRK eine Tendenz zur Öffnung: Zwar folgt aus den genannten Normen mit Blick auf die noch disparate Staatenpraxis kein Anspruch auf Einführung der Ehe für gleichgeschlechtliche Paare; führt ein Staat die gleichgeschlechtliche Ehe jedoch ein, unterfällt das Interesse Gleichgeschlechtlicher an einer Eheschließung dem Schutz des Art. 9 GrCh in Verbindung mit Art. 6 Abs. 1 EUV bzw. Art. 12 EMRK in Verbindung mit Art. 6 Abs. 3 EUV. ${ }^{136}$ Dagegen würde der EGMR ein voraussetzungsloses Recht auf Eheschließung (Öffnung der Ehe) aus Art. 12 EMRK allenfalls dann eines eher fernen Tages annehmen, wenn bereits eine überwältigende Mehrheit der Europaratsstaaten ${ }^{137}$ die Ehe für gleichgeschlechtliche Paare geöffnet haben sollte.

\section{Die Binnenmarktdimension}

Zum Anderen gibt es eine Binnenmarktdimension. Wir finden in Europa zurzeit noch ein System vor, indem die Mitgliedstaaten zwar für gleichgeschlechtliche Paare die Ehe öffnen oder die eingetragene Partnerschaft einführen können. Der Grundsatz der Einheitlichkeit der persönlichen Rechtsstellung führt dann auch dazu, dass dieser Personenstand europaweit akzeptiert werden muss. Ob die gleichgeschlechtlichen (Ehe-)Partner in anderen EULändern aber auch die für Ehepartner vorgesehenen Vergünstigungen erhalten, hängt von der Anerkennung gleichgeschlechtlicher Rechtsgemeinschaften im Empfangsstaat ab. Kennzeichnend für die Binnenmarktdimension ist dabei die fehlende Äquivalenz: Während das Vertrauens- oder Äquivalenzprinzip ${ }^{138}$ die Mitgliedstaaten der EU zwingt, ihre jeweiligen Regelungen zur Verkehrsfähigkeit von Waren oder zur Gründung von Handelsgesellschaften gegenseitig anzuerkennen, sich also hier auch schon im Bereich der Vorbedingungen für die Nutzbarkeit von Grundfreiheiten dem Wettbewerb der Systeme auszusetzen, soll die in einem Mitgliedstaat wirksam geschlossene „Ehe“ (insbesondere die gleichgeschlechtliche) nicht auch in jedem anderen EU-Land als Ehe behandelt werden müssen - obwohl erst deren Anerkennung den betroffenen Paaren den vollen Genuss der Personenverkehrsfreiheiten bzw. den gleichen Genuss im Vergleich zu verschiedengeschlechtlichen Ehepaaren verschaffen würde. Zugespitzt formuliert genießt die Ware oder die $\mathrm{GmbH}$ in Bezug auf ihren jeweiligen Grund-Status mehr Respekt als die (gleichgeschlechtliche) Person. Erklärungen hierfür sind zwar leicht zur Hand. Sie reichen von der

136 Siehe unter B.III. und C.II.

137 Zur Methode der Rechtsfortbildung von Ungern-Sternberg, Die Konsensmethode des EGMR Eine kritische Bewertung mit Blick auf das völkerrechtliche Konsens- und das innerstaatliche Demokratieprinzip, Archiv des Völkerrechts 51 (2013), S. 312 ff.

138 Siehe unter B.IV.2.a). 
Verschiedenartigkeit der Materien bis zur gegebenen Kompetenzlage nach den europäischen Verträgen. Das Herkunftsland- oder Vertrauensprinzip reicht in diesem Sinne nicht weiter als die Grundfreiheiten selbst. ${ }^{139}$ Und doch bleibt die Frage, ob sich ein hochintegriertes System wie die EU Nicht-Äquivalenz in Fragen des Personenstands auf Dauer leisten kann.

\section{Zukunftsszenario: Entstehung einer „Ehe zweiter Klasse“?}

Welchen Sinn haben eingetragene Partnerschaft und gleichgeschlechtliche Ehe im europäischen Binnenmarkt, wenn sie dort keine Folgewirkungen haben bzw. zu einer schlechteren Position als im Heimatstaat führen? Schon im Fall D. und Königreich Schweden ging es dem Beamten $D$. ja nicht nur darum, dass sein formaler Status der Verpartnerung unangetastet blieb, sondern auch darum, dass die mit diesem Status typischerweise verknüpften Rechtsfolgen anerkannt werden, wenn er eine europäische Grenze überschreitet oder für die Europäische Union arbeitet. Betroffen sind hier Fragen der Personenzügigkeit und der Funktionsfähigkeit des Binnenmarkts. Es überzeugt nicht, dass Ehepartner in allen europäischen Staaten darauf vertrauen können, dass ihnen die jeweils vorgesehenen Vergünstigungen für Ehegatten zuteil werden, während gleichgeschlechtliche Partner im schlechtesten Fall im Empfangsstaat wie Personen behandelt werden, die unverbindlich zusammenleben.

Ein vergleichbarer Gedanke liegt auch der jüngsten Entscheidung des U.S. Supreme Court zur Verfassungswidrigkeit des Defense of Marriage Act (DOMA 2013) ${ }^{140}$ zugrunde. Denn das umstrittene Bundesgesetz (DOMA 2013) hätte im Ergebnis zur Nichtanerkennung einzelstaatlich wirksam geschlossener gleichgeschlechtlicher Ehen im Anwendungsbereich des Bundesrechts geführt. Damit würden jedoch, so der Supreme Court, gleichgeschlechtliche Paare in die ,instabile Position einer zweitrangigen Ehe” versetzt; vielmehr: die Differenzierung erniedrige das Paar wie auch die Kinder, die in solchen Verbindungen lebten. ${ }^{141} \mathrm{Im}$ Vergleich hierzu verbietet das europäische Recht zwar keineswegs die gleichgeschlechtliche Ehe; es versagt den gleichgeschlechtlichen Ehepartnern aber jeden Anspruch darauf, die üblicherweise mit einer „Ehe“ verbundenen Vorteile auch in anderen Mitgliedstaaten zu genießen. Auch hierdurch kommt es zur ,instabilen Position einer

139 Kingreen, (Fn. 26), Art. 34-36 AEUV, Rdnr. 151. Zu eng allerdings die Annahme, dass es sich (nur) um eine Kollisionsregel handele (ibid.).

140 Act of September 21, 1996, 110 Stat. (Statutes at Large) 2419.

141 U.S. Supreme Court, 570 U.S. [...] (2013), Urt. v. 26.6.2013, www.supremecourt.gov/opinions/ 12pdf/12-307_6j37.pdf (25.6.2014), S. 22 f.: „By creating two contradictory marriage regimes within the same State, DOMA forces same-sex couples to live as married for the purpose of state law but unmarried for the purpose of federal law, thus diminishing the stability and predictability of basic personal relations the State has found it proper to acknowledge and protect. By this dynamic DOMA undermines both the public and private significance of state sanctioned samesex marriages; for it tells those couples, and all the world, that their otherwise valid marriages are unworthy of federal recognition. This places same-sex couples in an unstable position of being in a second-tier marriage. The differentiation demeans the couple, whose moral and sexual choices the Constitution protects, see [...] and whose relationship the State has sought to dignify. And it humiliates tens of thousands of children now being raised by same-sex couples." 
zweitrangigen Ehe“, sobald die Partner ihren Aufenthalt kraft der Binnenmarktfreiheiten in einem anderen Mitgliedstaat nehmen, der ihrer Ehe jede praktische Bedeutung nimmt.

Zumindest im Bereich des EU-Sekundärrechts lassen neue Richtlinien, die ungeachtet der Existenz gleichgeschlechtlicher Ehen in zunehmend mehr Mitgliedstaaten weiterhin nur zwischen „Ehegatten“ und „Lebenspartnern“ unterscheiden, den Schluss zu, dass alle Personen Ehegatten sind, die diesen Status in irgendeinem EU-Mitgliedstaat wirksam erworben haben - sei es auf der Basis der Verschieden- oder aber der Gleichgeschlechtlichkeit. ${ }^{142}$ Will der EU-Gesetzgeber diese Konsequenz vermeiden bzw. den Mitgliedstaaten Raum zur Versagung der Eheprivilegien geben, muss er in den betreffenden Richtlinien die ,gleichgeschlechtliche Ehe“" neben der Lebenspartnerschaft ausdrücklich benennen und beide offen erkennbar von der verschiedengeschlechtlichen Ehe trennen. Dann würde allerdings auch in Europa die Existenz einer „Ehe zweiter Klasse“ sichtbar werden. Es ist nicht die Anerkennung eines verschwindend geringen Anteils gleichgeschlechtlicher Ehen, sondern die drohende Mehrdeutigkeit des Konzepts „Ehe“ durch Nichtanerkennung wirksam geschlossener gleichgeschlechtlicher Ehen, welche die besondere Institution „Ehe“ am allermeisten schädigen wird. 